\title{
ON THE HARTSHORNE-HIRSCHOWITZ THEOREM
}

\author{
T. ALADPOOSH AND M. V. CATALISANO
}

\begin{abstract}
The Hartshorne-Hirschowitz theorem says that a generic union of lines in $\mathbb{P}^{n},(n \geq 3)$, has good postulation. The proof of Hartshorne and Hirschowitz in the initial case $\mathbb{P}^{3}$ is difficult and so long, which is handled by a method of specialization via a smooth quadric surface with the property of having two rulings of skew lines. We provide a proof in the case $\mathbb{P}^{3}$ based on a new degeneration of disjoint lines via a plane $H \cong \mathbb{P}^{2}$, which we call $(2, s)$-cone configuration, that is a schematic union of $s$ intersecting lines passing through a single point $P$ together with the trace of an $s$-multiple point supported at $P$ on the double plane $2 H$. In the first part of this paper, we discuss our degeneration inductive approach. We prove that a $(2, s)$-cone configuration is a degeneration of $s$ disjoint lines in $\mathbb{P}^{3}$, or more generally in $\mathbb{P}^{n}$. In the second part of the paper, we use this degeneration in an effective method to show that a generic union of lines in $\mathbb{P}^{3}$ imposes independent conditions on the linear system $\left|\mathcal{O}_{\mathbb{P}^{3}}(d)\right|$ of surfaces of given degree $d$. The basic motivation behind our degeneration approach is that it looks more systematic that gives some hope of extensions to the analogous problem in higher dimensional spaces, that is the postulation problem for $m$-dimensional planes in $\mathbb{P}^{2 m+1}$.
\end{abstract}

\section{Contents}

1. Introduction

2. Preliminaries and Notations 4

3. Main Argument via Degeneration; $(2, s)$-Cone Configuration

3.1. Definitions and Basic Constructions 8

3.2. An Example: The Case of $(2,3)$-Cone Configuration 10

3.3. A Degeneration Inductive Method: How $(2, \mathrm{~s})$-Cone Configurations Appear as Degenerations of $s$ Skew Lines? 13

4. Reduction Process 19

4.1. Auxiliary Vanishing Statements 19

4.2. $\quad \mathbf{H}_{\mathbf{d}-\mathbf{1}}^{\prime}+\mathbf{H}_{\mathbf{d}}^{\prime \prime} \Rightarrow \mathbf{H}_{\mathbf{d}}$ for $\mathbf{d} \geq \mathbf{3}$

5. Proof of $\mathbf{H}_{d-1}^{\prime} \Rightarrow 22$

2010 Mathematics Subject Classification. 14N20, 14C20, 14D06, 13D40.

Keywords: Good postulation; $(2, s)$-Cone configuration; Degeneration; Specialization; Generic lines. 
5.1. Case $\mathbf{d} \equiv \mathbf{0}(\bmod 3) \quad 24$

5.2. Case $\mathbf{d} \equiv \mathbf{1}(\bmod 3) \quad 26$

5.3. Case $\mathbf{d} \equiv \mathbf{2}(\bmod 3) \quad 29$

6. Proof of $\mathbf{H}_{d}^{\prime \prime} \quad 31$

6.1. Case $\mathbf{d} \equiv \mathbf{0}, \mathbf{1}(\bmod 3)$

6.2. Case $\mathbf{d} \equiv \mathbf{2}(\bmod 3) \quad 32$

References 33

\section{INTRODUCTION}

Given a closed subscheme $X \subset \mathbb{P}^{n}$, we say that $X$ has good postulation or maximal rank if $X$ imposes the expected number of conditions to hypersurfaces of any degree. This is equivalent to saying that for each $d \geq 0$ one or other of the integers $h^{0}\left(\mathcal{I}_{X}(d)\right), h^{1}\left(\mathcal{I}_{X}(d)\right)$ is zero. This problem is equivalent to computing the Hilbert function of $X$. Let $H F(X, d)$ be the Hilbert function of $X$ in degree $d$, namely, $H F(X, d)=h^{0}\left(\mathcal{O}_{\mathbb{P}^{n}}(d)\right)-h^{0}\left(\mathcal{I}_{X}(d)\right)$. There is an expected value for the Hilbert function of $X$ in degree $d$ given by a naive count of conditions. This value is determined by assuming that $X$ imposes independent conditions on the linear system $\left|\mathcal{O}_{\mathbb{P}^{n}}(d)\right|$, i.e.,

$$
h^{0}\left(\mathcal{I}_{X}(d)\right)=\max \left\{h^{0}\left(\mathcal{O}_{\mathbb{P}^{n}}(d)\right)-h^{0}\left(\mathcal{O}_{X}(d)\right), 0\right\},
$$

which implies that $X$ has good postulation in degree $d$.

When we restrict our attention to the special class of schemes $X \subset \mathbb{P}^{n}$ which supported on unions of generic linear spaces there is much interest in the postulation problem (see e.g. [GMR83], [HH81], [CCG10], [Bal11] for reduced case, and [CCG16], [AB14], [Bal15], [Ala16], [BDS $\left.{ }^{+} 17\right]$ for non-reduced case), yet surprisingly very little is known about them, even in the reduced case. Specifically concerning the class of reduced schemes of generic linear spaces, the first obvious case is to take $X$ a generic collection of points in $\mathbb{P}^{n}$, according to [GMR83] it is well known that $X$ has good postulation. In the next case concerning a generic collection of lines in $\mathbb{P}^{n}$, there is a spectacular theorem by R. Hartshorne and A. Hirschowitz [HH81], going back to 1981, which states that:

Theorem 1.1 (Hartshorne-Hirschowitz). Let $X \subset \mathbb{P}^{n},(n \geq 3)$, be a generic union of e lines. Then $X$ has good postulation, i.e.,

$$
h^{0}\left(\mathcal{I}_{X}(d)\right)=\max \left\{\left(\begin{array}{c}
d+n \\
n
\end{array}\right)-e(d+1), 0\right\} .
$$

Inspired by these results about points and skew lines, E. Carlini, M. V. Catalisano and A. V. Geramita proposed a conjecture on the postulation of generic disjoint unions of linear spaces [CCG10, §1], which says that: 
Conjecture 1.2 (Carlini-Catalisano-Geramita). If $X \subset \mathbb{P}^{n}$ is a generic union of linear spaces with non-intersecting components, then $X$ has good postulation.

As we have mentioned above, this conjecture is true for $\operatorname{dim} X=0$, where only points are involved, and for $\operatorname{dim} X=1$, i.e. a generic collection of lines and points, where we have Hartshorne-Hirschowitz theorem about generic lines and also we know how adding generic points to a scheme can still preserve its good postulation [GMR83]. As soon as we go up to $\operatorname{dim} X>1$, the problem becomes more and more complicated. In fact, when $\operatorname{dim} X>1$ the results in the literature are so little and the conjecture remains widely open even for $\operatorname{dim} X=2$ (see e.g. [CCG10] and [Bal11] for a generic union of lines and a few planes).

The basic natural step in the proof of Conjecture 1.2 for $\operatorname{dim} X>1$, would be to provide an analogue of Hartshorne-Hirschowitz theorem for a generic collection of planes in $\mathbb{P}^{n},(n \geq 5)$, that seems to be extremely difficult, and even surprisingly enough, one may hope to generalize this approach to the case of $m$-dimensional planes, $\left(m\right.$-planes for short), in $\mathbb{P}^{n},(n \geq 2 m+1)$. Actually, the postulation problem for $m$-planes is so difficult that one seldom expects to solve it completely, yet which provides stimulus for a great amount of efforts, and which enable us to make progress on this problem.

Nevertheless, if we wish to go further in this direction we need to analyze the proof of Hartshorne and Hirschowitz. Interestingly enough, their proof in the initial case $\mathbb{P}^{3}$ is difficult and long by using degeneration techniques via a smooth quadric surface, which occupies more than half of the length of the paper [HH81]. Indeed, one aspect of the results in their paper is the postulation of degenerated schemes, which plays an important role in the proof of their main theorem (Theorem 1.1). Roughly speaking, what we mean by degeneration of a scheme, is a limiting scheme inside a projective space of a flat family of original ones. Apart from the fact that, as $X$ varies in a flat family, by the semicontinuity theorem for cohomology groups [Har77, III, 12.8], the condition of good postulation is an open condition on the family of $X$, one may use degenerations and the semicontinuity theorem to investigate that $X$ has good postulation. This means that, to prove that $X$ has good postulation it is enough to find a degeneration of $X$ which has good postulation. It is the degeneration that requires some artistry and a lot of technical details, which usually gives the most problems. It is precisely in this part of the procedure that we will give some new ideas. In fact, the main goal of this paper is: firstly introducing a new degeneration of $s$ disjoint lines in $\mathbb{P}^{3}$ (or even in $\left.\mathbb{P}^{n}\right)$, which we will call $(2, s)$-cone configuration, and secondly verifying the postulation of generic unions of lines by applying successfully this method of degeneration. An important feature of the $(2, s)$-cone configuration is that it 
will be extremely useful when we will attack the postulation problem of generic lines in $\mathbb{P}^{3}$ using degenerations via a $\mathbb{P}^{2}$ instead of the smooth quadric surface.

The paper is organized as follows. Section 2 contains preliminary material. In Section 3, we first introduce the notion of $(2, s)$-cone configuration (see $\S 3.1)$; next we show that a $(2, s)$-cone configuration is a flat limit of a family of disjoint unions of $s$ lines, that is a degeneration of $s$ skew lines (see $\S 3.2$, specially Figure 1, for the case $s=3$ and $\S 3.3$ for the general case). In Section 4, with the purpose of stating a proof of Hartshorne-Hirschowitz theorem in the case of $\mathbb{P}^{3}$ using this new method of degeneration, we reformulate Theorem 1.1 to a good postulation statement $\mathbf{H}_{d}$ (see $\S 4.1$ ); then we propose two other good postulation statements $\mathbf{H}_{d-1}^{\prime}$ and $\mathbf{H}_{d}^{\prime \prime}$, which are necessary for our inductive approach (see §4.2). Finally Sections 5, 6 give the proofs of the statements $\mathbf{H}_{d-1}^{\prime}$ and $\mathbf{H}_{d}^{\prime \prime}$, respectively.

We would like to finish Introduction by mentioning that, with an eye towards handling the postulation problems of disjoint unions of $m$-planes in $\mathbb{P}^{n}$ $(n \geq 2 m+1)$, one motivation for us comes from the fact about the lack of hypersurfaces in the initial case $\mathbb{P}^{2 m+1}$ with geometric constructions analogue to the smooth quadric surface in $\mathbb{P}^{3}$; while hyperplanes have constructions analogue to the plane. This leads us to provide a proof of Hartshorne-Hirschowitz theorem in $\mathbb{P}^{3}$ using degenerations by a plane instead of the quadric surface. Actually, in analogy with the degeneration of $s$ disjoint lines, we believe that the notion of $(2, s)$-cone configuration can be extended, somehow, for $s$ disjoint $m$-planes, which appears to be ambitious and requires the most sophisticated investigations, and then one may hope to generalize our approach to the postulation problem of $m$-planes.

\section{Preliminaries And Notations}

In this paper we work over an algebraically closed field $k$ with characteristic zero.

Given a closed subscheme $X$ of $\mathbb{P}^{n}, I_{X}$ and $\mathcal{I}_{X}$ will denote the homogeneous ideal and the ideal sheaf of $X$, respectively.

If $X, Y$ are closed subschemes of $\mathbb{P}^{n}$ and $X \subset Y$, then we denote by $\mathcal{I}_{X, Y}$ the ideal sheaf of $X$ in $\mathcal{O}_{Y}$.

If $X$ and $Y$ are two closed subschemes of $\mathbb{P}^{n}$, we denote by $X+Y$ the schematic union of $X$ and $Y$, i.e. the subscheme of $\mathbb{P}^{n}$ defined by the ideal sheaf $\mathcal{I}_{X} \cap \mathcal{I}_{Y} \subset \mathcal{O}_{\mathbb{P}^{n}}$.

If $\mathcal{F}$ is a coherent sheaf on the scheme $X$, for any integer $i \geq 0$ we use $h^{i}(X, \mathcal{F})$ to denote the $k$-vector space dimension of the cohomology group $H^{i}(X, \mathcal{F})$. In particular, when $X=\mathbb{P}^{n}$, we will often omit $X$ and we will simply write $h^{i}(\mathcal{F})$. 
The basic tool for the study of the postulation problem is the so called Castelnuovo's inequality (for proof we refer to [AH95, Section 2] or [AH00]).

We first recall the notion of residual scheme [Ful84, §9.2.8].

Definition 2.1. Let $X, Y$ be closed subschemes of $\mathbb{P}^{n}$.

(i) The closed subscheme of $\mathbb{P}^{n}$ defined by the ideal sheaf $\left(\mathcal{I}_{X}: \mathcal{I}_{Y}\right)$ is called the residual of $X$ with respect to $Y$ and denoted by $\operatorname{Res}_{Y}(X)$.

(ii) The schematic intersection $X \cap Y$ defined by the ideal sheaf $\left(\mathcal{I}_{X}+\right.$ $\left.\mathcal{I}_{Y}\right) / \mathcal{I}_{Y}$ of $\mathcal{O}_{Y}$ is called the trace of $X$ on $Y$ and denoted by $\operatorname{Tr}_{Y}(X)$.

We note that the generally valid identity for ideal sheaves

$$
\left(\mathcal{I}_{X_{1}} \cap \mathcal{I}_{X_{2}}: \mathcal{I}_{Y}\right)=\left(\mathcal{I}_{X_{1}}: \mathcal{I}_{Y}\right) \cap\left(\mathcal{I}_{X_{2}}: \mathcal{I}_{Y}\right)
$$

implies that the residual of the schematic union $X_{1}+X_{2}$ is the schematic union of the residuals.

Lemma 2.2 (Castelnuovo's Inequality). Let $d, e \in \mathbb{N}$, and $d \geq e$. Let $H \subseteq \mathbb{P}^{n}$ be a hypersurface of degree $e$, and let $X \subseteq \mathbb{P}^{n}$ be a closed subscheme. Then

$$
h^{0}\left(\mathbb{P}^{n}, \mathcal{I}_{X}(d)\right) \leq h^{0}\left(\mathbb{P}^{n}, \mathcal{I}_{\operatorname{Res}_{H}(X)}(d-e)\right)+h^{0}\left(H, \mathcal{I}_{\operatorname{Tr}_{H}(X)}(d)\right) .
$$

The following remark is quite immediate.

Remark 2.3. Let $X=X_{1}+\cdots+X_{s} \subset \mathbb{P}^{n}$ be the union of non-intersecting closed subschemes $X_{i}$. Let $s^{\prime}<s$ and

$$
X^{\prime}=X_{1}+\cdots+X_{s^{\prime}} \subset X .
$$

(i) If $h^{1}\left(\mathcal{I}_{X}(d)\right)=0$, then $h^{1}\left(\mathcal{I}_{X^{\prime}}(d)\right)=0$.

(ii) If $h^{0}\left(\mathcal{I}_{X^{\prime}}(d)\right)=0$, then $h^{0}\left(\mathcal{I}_{X}(d)\right)=0$.

A (fat) point of multiplicity $m$, or an m-multiple point, with support $P \in \mathbb{P}^{n}$, denoted $m P$, is the zero-dimensional subscheme of $\mathbb{P}^{n}$ defined by the ideal sheaf $\left(\mathcal{I}_{P}\right)^{m}$, i.e. the $(m-1)^{t h}$ infinitesimal neighborhood of $P$. In particular, if $m=2$ we shall call the scheme $2 P$ a double point with support $P$, i.e. the first infinitesimal neighborhood of $P$.

In case $P \in X$ for any smooth variety $X \subset \mathbb{P}^{n}$, we will write $\left.m P\right|_{X}$ for the $(m-1)^{t h}$ infinitesimal neighborhood of $P$ in $X$, that is the schematic intersection of the $m$-multiple point $m P$ of $\mathbb{P}^{n}$ and $X$ with $\left(\mathcal{I}_{P, X}\right)^{m}$ as its ideal sheaf.

More generally, if $\mathbb{X}=\left\{P_{1}, \ldots, P_{s}\right\}$ is any set of distinct points in $\mathbb{P}^{n}$ and $m_{1}, \ldots, m_{s}$ are positive integers, then we will denote by $m_{1} P_{1}+\cdots+m_{s} P_{s}$ the zero-dimensional subscheme of $\mathbb{P}^{n}$ defined by the ideal sheaf $\left(\mathcal{I}_{P_{1}}\right)^{m_{1}} \cap \ldots \cap$ $\left(\mathcal{I}_{P_{s}}\right)^{m_{s}}$, and we sometimes refer to it as a fat point scheme with support $\mathbb{X}$.

We recall a spectacular result due to Alexander and Hirschowitz, on the postulation of generic collections of double points. 
Theorem 2.4 (Alexander-Hirschowitz). [AH95] Let $n, d \in \mathbb{N}$, and let $X \subset \mathbb{P}^{n}$ be a generic collection of $s$ double points. Then $X$ has good postulation in degree $d$ except in the following cases:

- $d=2,2 \leq s \leq n$;

- $n=2, d=4, s=5$;

- $n=3, d=4, s=9$;

- $n=4, d=3, s=7$;

- $n=4, d=4, s=14$.

Since we will apply Alexander-Hirschowitz theorem in the case of $\mathbb{P}^{2}$ frequently in Section 6, it is convenient to restate it as follows.

Corollary 2.5. The scheme $X \subset \mathbb{P}^{2}$ consisting of s generic double points always has good postulation in degree $d$, except for the two cases $\{s=2, d=2\}$ and $\{s=5, d=4\}$.

If $X$ is a zero-dimensional scheme, we denote by $\ell(X)$ its length. So in the simple case $X=m P \subset \mathbb{P}^{n}$ we have $\ell(X)=\left(\begin{array}{c}n+m-1 \\ n\end{array}\right)$.

Even though in this paper we will only use 2-dots, it seemed nice to give the more general definition as follows: a $\delta$-dot is a subscheme of length $\delta$ of a double point. (Hence a double point in $\mathbb{P}^{n}$ is an $(n+1)$-dot.)

From [CG94] we know that a generic union of 2-dots in $\mathbb{P}^{2}$ has good postulation. Now the next lemma gives more information on the postulation problem regarding 2-dots in $\mathbb{P}^{2}$. Precisely the lemma affirms that a generic union of 2-dots together with a multiple point in $\mathbb{P}^{2}$ has good postulation, which geometrically means that a generic collection of 2-dots imposes the expected number of conditions on the linear system of curves passing through a multiple point.

Lemma 2.6. Let $s, m, d \in \mathbb{N}$, and $d \geq m-1$. Let the scheme $X \subset \mathbb{P}^{2}$ be a generic union of an $m$-multiple point $m P$ and $s 2$-dots $Z_{1}, \ldots, Z_{s}$. Then $X$ has good postulation, i.e.

$$
h^{0}\left(\mathcal{I}_{X}(d)\right)=\max \left\{\left(\begin{array}{c}
d+2 \\
2
\end{array}\right)-\left(\begin{array}{c}
m+1 \\
2
\end{array}\right)-2 s, 0\right\},
$$

Proof. Let

$$
t=\left\lfloor\frac{\left(\begin{array}{c}
d+2 \\
2
\end{array}\right)-\left(\begin{array}{c}
m+1 \\
2
\end{array}\right)}{2}\right\rfloor ; \quad u=\left\lceil\frac{\left(\begin{array}{c}
d+2 \\
2
\end{array}\right)-\left(\begin{array}{c}
m+1 \\
2
\end{array}\right)}{2}\right\rceil .
$$

In order to apply Remark 2.3, we must prove the lemma only for the two cases $s=t$ and $s=u$.

If $\left(\begin{array}{c}d+2 \\ 2\end{array}\right)-\left(\begin{array}{c}m+1 \\ 2\end{array}\right)$ is even, we have $t=u$. If $\left(\begin{array}{c}d+2 \\ 2\end{array}\right)-\left(\begin{array}{c}m+1 \\ 2\end{array}\right)$ is odd, we observe that for $s=t$ the expected value for $h^{0}\left(\mathcal{I}_{X}(d)\right)$ is 1 . So, if we prove that 
$h^{0}\left(\mathcal{I}_{X}(d)\right)=1$ for $s=t$, we then conclude that $h^{0}\left(\mathcal{I}_{X}(d)\right)=0$ for $s=u$, i.e. the expected one. Therefore it is enough to prove the lemma only for $s=t$.

If $m=0$, the scheme $X$ is generic union of 2-dots, and the conclusion immediately follows from [CG94, Proposition 4.2].

Now we assume that $m>0$. We will proceed by induction on $m$.

Let $L$ be a line passing through the point $P$. We consider two cases.

Case 1. When $d-m$ is odd. Let $t^{\prime}=\frac{d-m+1}{2}$, (note that $t \geq t^{\prime}$ ). Consider the scheme $\widetilde{X}$ obtained from $X$ by specializing the 2 -dots $Z_{1}, \ldots, Z_{t^{\prime}}$ so that $Z_{i} \subset L,\left(1 \leq i \leq t^{\prime}\right)$, (the 2 -dots $Z_{t^{\prime}+1}, \ldots, Z_{t}$ remain generic not lying on $L$ ).

Since $\operatorname{deg}(\tilde{X} \cap L)=m+2 t^{\prime}=d+1$, the line $L$ is a fixed component for the curves of degree $d$ containing $\tilde{X}$, so we get

$$
h^{0}\left(\mathcal{I}_{\widetilde{X}}(d)\right)=h^{0}\left(\mathcal{I}_{\operatorname{Res}_{L}(\widetilde{X})}(d-1)\right)
$$

where $\operatorname{Res}_{L}(\widetilde{X})$ is the union of the $(m-1)$-multiple point $(m-1) P$ and 2-dots $Z_{t^{\prime}+1}, \ldots, Z_{t}$. Thus by the induction hypothesis we have

$$
\begin{aligned}
h^{0}\left(\mathcal{I}_{\operatorname{Res}_{L}(\widetilde{X})}(d-1)\right) & =\max \left\{\left(\begin{array}{c}
d+1 \\
2
\end{array}\right)-\left(\begin{array}{c}
m \\
2
\end{array}\right)-2\left(t-t^{\prime}\right), 0\right\} \\
& =\max \left\{\left(\begin{array}{c}
d+2 \\
2
\end{array}\right)-\left(\begin{array}{c}
m+1 \\
2
\end{array}\right)-2 t, 0\right\},
\end{aligned}
$$

which by the equality (1) gives

$$
h^{0}\left(\mathcal{I}_{\widetilde{X}}(d)\right)=\max \left\{\left(\begin{array}{c}
d+2 \\
2
\end{array}\right)-\left(\begin{array}{c}
m+1 \\
2
\end{array}\right)-2 t, 0\right\},
$$

and from here, by semicontinuity of cohomology [Har77, III, 12.8], we see that

$$
h^{0}\left(\mathcal{I}_{X}(d)\right)=\max \left\{\left(\begin{array}{c}
d+2 \\
2
\end{array}\right)-\left(\begin{array}{c}
m+1 \\
2
\end{array}\right)-2 t, 0\right\}
$$

hence this case is done.

Case 2. When $d-m$ is even. Let $t^{\prime}=\frac{d-m}{2}$, (note that $t \geq t^{\prime}+1$ ). We specialize the 2 -dots $Z_{1}, \ldots, Z_{t^{\prime}}$ so that $Z_{i} \subset L,\left(1 \leq i \leq t^{\prime}\right)$; moreover, we specialize $Z_{t^{\prime}+1}$ in such a way that the support of $Z_{t^{\prime}+1}$ is contained in the line $L$, but $Z_{t^{\prime}+1} \not \subset L$, which implies that $\operatorname{deg}\left(Z_{t^{\prime}+1} \cap L\right)=1$. Let $\widetilde{X}$ be the specialized scheme, (note that the 2 -dots $Z_{t^{\prime}+2}, \ldots, Z_{t}$ remain generic not lying on $L)$.

Since $\operatorname{deg}(\tilde{X} \cap L)=m+2 t^{\prime}+1=d+1$, the line $L$ is a fixed component for the curves of degree $d$ containing $\tilde{X}$, so we get

$$
h^{0}\left(\mathcal{I}_{\widetilde{X}}(d)\right)=h^{0}\left(\mathcal{I}_{\operatorname{Res}_{L}(\widetilde{X})}(d-1)\right)
$$


where, by noting that $\operatorname{Res}_{L}\left(Z_{t^{\prime}+1}\right)$ is a simple point, the scheme $\operatorname{Res}_{L}(\widetilde{X})$ is the generic union of the $(m-1)$-multiple point $(m-1) P$, 2-dots $Z_{t^{\prime}+2}, \ldots, Z_{t}$ and one simple point. Hence by the induction hypothesis we have

$$
\begin{aligned}
h^{0}\left(\mathcal{I}_{\operatorname{Res}_{L}(\tilde{X})}(d-1)\right) & =\max \left\{\left(\begin{array}{c}
d+1 \\
2
\end{array}\right)-\left(\begin{array}{c}
m \\
2
\end{array}\right)-2\left(t-t^{\prime}-1\right)-1,0\right\} \\
& =\max \left\{\left(\begin{array}{c}
d+2 \\
2
\end{array}\right)-\left(\begin{array}{c}
m+1 \\
2
\end{array}\right)-2 t, 0\right\},
\end{aligned}
$$

then by the equality (2) and the semicontinuity of cohomology [Har77, III, 12.8] we get the conclusion

$$
h^{0}\left(\mathcal{I}_{X}(d)\right)=\max \left\{\left(\begin{array}{c}
d+2 \\
2
\end{array}\right)-\left(\begin{array}{c}
m+1 \\
2
\end{array}\right)-2 t, 0\right\},
$$

which finishes the proof.

\section{Main Argument via Degeneration;}

$(2, s)$-Cone Configuration

A natural approach to the postulation problem is to argue by degeneration. In view of the fact that we have the semicontinuity theorem for cohomology groups in a flat family [Har77, III, 12.8], one may use the degenerations and the semicontinuity theorem in order to be able to better handle the postulation of schemes supported on generic unions of linear spaces. Specifically, if one can prove that the property of having good postulation is satisfied in the special fiber, i.e. the degenerate scheme, then one may hope to obtain the same property in the general fiber, i.e. the original scheme.

3.1. Definitions and Basic Constructions. In the celebrated paper [HH81] Hartshorne and Hirschowitz investigated a new degeneration technique to attack the postulation problem for a generic union of lines. In fact, they degenerate two skew lines in $\mathbb{P}^{3}$ in such a way that the resulting scheme becomes a "degenerate conic with an embedded point" (which also was used in [Hir81]). Even more generally, one can push this trick of "adding nilpotents" further, to give a degeneration of two skew lines in higher dimensional projective spaces $\mathbb{P}^{n}, n \geq 3$, this is what the authors introduced in [CCG10, Definition 2.7 with $m=1]$ and called a (3-dimensional) sundial.

According to the terminology of [HH81], we say that $C$ is a degenerate conic if $C$ is the union of two intersecting lines $L$ and $M$, so $C=L+M$.

Now we recall the definition of a 3-dimensional sundial or simply a sundial (see [CCG11, Definition 3.7] or [CCG10, Definition 2.7 with $m=1$ ]).

Definition 3.1. Let $L$ and $M$ be two intersecting lines in $\mathbb{P}^{n}, n \geq 3$, and let $T \cong \mathbb{P}^{3}$ be a generic linear space containing the degenerate conic $L+M$. 
Let $P$ be the singular point of $L+M$, i.e. $P=L \cap M$. We call the scheme $L+M+\left.2 P\right|_{T}$ a degenerate conic with an embedded point or a (3-dimensional) sundial.

One can show a sundial is a flat limit inside $\mathbb{P}^{n}$ of a flat family whose general fiber is the disjoint union of two lines, i.e. a sundial is a degeneration of two generic lines in $\mathbb{P}^{n}, n \geq 3$. This is the content of the following lemma (see [HH81, Example 2.1.1] for the case $n=3$, and [CCG11, Lemma 3.8] or [CCG10, Lemma 2.5 with $m=1$ ] for the general case $n \geq 3$ ).

Lemma 3.2. Let $\mathcal{X}_{1} \subset \mathbb{P}^{n}, n \geq 3$, be the disjoint union of two lines $L_{1}$ and M. Then there exists a flat family of subschemes $\mathcal{X}_{\lambda} \subset\left\langle\mathcal{X}_{1}\right\rangle \cong \mathbb{P}^{3},(\lambda \in k)$, whose general fiber is the union of two skew lines and whose special fiber is the sundial $\mathcal{X}_{0}=M+L+\left.2 P\right|_{\left\langle\mathcal{X}_{1}\right\rangle}$, where $L$ is a line and $M \cap L=P$.

Definition 3.3. A $(2, m ; n)$-point is a zero-dimensional scheme in $\mathbb{P}^{n}, n \geq 3$, with support at one point $P$, and whose ideal sheaf is of type $\mathcal{I}_{P}^{m}+\mathcal{I}_{H}^{2}$, where $H \subset \mathbb{P}^{n}$ is a plane containing $P$. We denote it by $\mathfrak{D}_{H, m}(P)$, or $\mathfrak{D}_{m}(P)$ if no confusion arises. So $\mathfrak{D}_{H, m}(P)$ is the trace of $m P$ on the double plane $2 H$,

$$
\mathfrak{D}_{H, m}(P)=m P \cap 2 H .
$$

If we take the residual and the trace of the $(2, m ; n)$-point $\mathfrak{D}_{H, m}(P)$ with respect to $H$ we observe that the residual scheme $\operatorname{Res}_{H}\left(\mathfrak{D}_{H, m}(P)\right)$ is the subscheme $\left.(m-1) P\right|_{H}$ of $\mathbb{P}^{n}$, that is $(m-2)^{t h}$ infinitesimal neighborhood of $P$ in $H$, and the trace scheme $\operatorname{Tr}_{H}\left(\mathfrak{D}_{H, m}(P)\right)$ is the $m$-multiple point $m P$ of $H$.

Definition 3.4. We say that $\mathcal{C}$ is a cone configuration of type $s, s \geq 3$, if $\mathcal{C}$ is the union of $s$ intersecting lines $L_{1}, \ldots, L_{s}$ passing through a single point $P$, so $\mathcal{C}=L_{1}+\cdots+L_{s}$ and $L_{1} \cap \ldots \cap L_{s}=P$.

With the help of Definitions 3.3 and 3.4, we make the following definition, which is essential to our degeneration approach and is central to the proof of our main theorem in Section 4.

Definition 3.5. Let $L_{1}, \ldots, L_{s}$ be $s$ lines in $\mathbb{P}^{n}$ lying on a plane and intersecting in a single point $P$, i.e. $L_{1} \cap \ldots \cap L_{s}=P$, and $n, s \geq 3$. Let $H \cong \mathbb{P}^{2}$ be the plane containing the cone configuration $L_{1}+\cdots+L_{s}$. We call the scheme $L_{1}+\cdots+L_{s}+\mathfrak{D}_{H, s}(P)$ a cone configuration of type $s$ with a $(2, s ; n)$-point or simply a $(2, s)$-cone configuration.

Then for the $(2, s)$-cone configuration $L_{1}+\cdots+L_{s}+\mathfrak{D}_{H, s}(P)$ the residual with respect to $H$ will be $\left.(s-1) P\right|_{H}$, while the trace with respect to $H$ will be the cone configuration $L_{1}+\cdots+L_{s}$.

Our interest in cone configurations, particularly $(2, s)$-cone configurations, arises from the important fact that these constructions are occurring as resulting schemes of specific degenerations of disjoint lines; however not in a 
straightforward way but rather in a less obvious and subtle way. This feature of $(2, s)$-cone configurations is interesting and surprising in its own and plays a key role in this paper.

Notation 3.6. Let $L_{1}, \ldots, L_{s}$ be $s \geq 2$ lines in $\mathbb{P}^{2}$ such that no three lines meet in a point. The set of $\left(\begin{array}{l}s \\ 2\end{array}\right)$ points which are the pairwise intersections of the lines $L_{i}$ is called a star configuration of points. We will also call the scheme $L_{1}+\cdots+L_{s}$ a star configuration of lines.

3.2. An Example: The Case of $(\mathbf{2}, \mathbf{3})$-Cone Configuration. If we consider in $\mathbb{P}^{3}$ a flat family of subschemes whose general scheme is the disjoint union of three lines, while the three lines meet at one single point in a special fiber, then the special scheme consists of three intersecting lines together with a $(2,3)$-point, i.e. a $(2,3)$-cone configuration. That is a remarkable result which we verify in this example.

Let $L_{1}, L_{2}, L_{3}$ be three disjoint lines in $\mathbb{P}^{3}$, and let $H$ be a fixed plane containing the line $L_{1}$. First We degenerate the lines $L_{2}, L_{3}$ in such a way that they together with $L_{1}$ become 3 lines in a star configuration in $H$. We will again denote these specialized lines by $L_{2}, L_{3}$, moreover, denote by $P, Q, R$ the three intersection points of the 3 lines $L_{1}, L_{2}, L_{3}$, namely

$$
P=L_{1} \cap L_{2} ; \quad Q=L_{1} \cap L_{3} ; \quad R=L_{2} \cap L_{3} .
$$

So according to Lemma 3.2 we get the star configuration $L_{1}+L_{2}+L_{3}$ in $H$ together with three double points, i.e. the specialized scheme is

$$
L_{1}+L_{2}+L_{3}+2 P+2 Q+2 R \subset \mathbb{P}^{3},
$$

which we call $\mathcal{X}_{1}$ (see Figure 1 ).

Now for the calculation. Choose coordinates $t, x, y, z$ on $\mathbb{P}^{3}$. We may assume that $P=[1: 0: 0: 0] ; Q=[1: 1: 0: 0] ; R=[1: 0: 1: 0]$, and the plane $H$ is defined by the equation $z=0$ (we can always choose coordinates so that this is the case). This implies that

$$
\left\{\begin{array} { l } 
{ I _ { P } = ( x , y , z ) ; } \\
{ I _ { Q } = ( x - t , y , z ) ; } \\
{ I _ { R } = ( y - t , x , z ) . }
\end{array} \quad \left\{\begin{array}{l}
I_{L_{1}}=(y, z) \\
I_{L_{2}}=(x, z) \\
I_{L_{3}}=(x+y-t, z) .
\end{array}\right.\right.
$$

Up to now, we found that the scheme $\mathcal{X}_{1}=L_{1}+L_{2}+L_{3}+2 P+2 Q+2 R$, which is a star configuration of 3 lines having the double structure at their intersection points, is a degeneration of three disjoint lines in $\mathbb{P}^{3}$. Next we want to make a further degeneration, precisely the degeneration of $\mathcal{X}_{1}$ in which the points $Q=[1: 1: 0: 0]$ and $R=[1: 0: 1: 0]$ move to the point $P=[1: 0: 0: 0]$. Now what is the resulting scheme of this degeneration? To answer this, let the line $L_{3}$ move in such a way that the two points $Q$ and $R$ approach the point $P$. We will show that this movement occurs in a flat family $\left\{\mathcal{X}_{\lambda}\right\}$ and the limiting 
scheme $\mathcal{X}_{0}$ is a cone configuration of type 3 union the $(2,3)$-point $3 P \cap 2 H$ (Figure 1). Then we can realize the limiting scheme as the scheme formed by $L_{1}+L_{2}+2 P$ and the limit of $L_{3}+2 Q+2 R$ along with the aforementioned direction.

We will now calculate the flat family $\left\{\mathcal{X}_{\lambda}\right\}_{\lambda \in k}$ just described, whereas $\mathcal{X}_{\lambda}$ is the scheme

$$
L_{1}+L_{2}+L_{3, \lambda}+2 P+2 Q_{\lambda}+2 R_{\lambda}
$$

where the two points $Q_{\lambda}=[1: \lambda: 0: 0]$ and $R_{\lambda}=[1: 0: \lambda: 0]$ move to the point $P=[1: 0: 0: 0]$, as well as the line joining $L_{3, \lambda}$, when $\lambda$ tends to 0 , that is

$$
\left\{\begin{array}{l}
I_{Q_{\lambda}}=(x-\lambda t, y, z) ; \\
I_{R_{\lambda}}=(y-\lambda t, x, z) ; \\
I_{L_{3, \lambda}}=(x+y-\lambda t, z) .
\end{array}\right.
$$

Then the homogeneous ideal of $\mathcal{X}_{\lambda}$ is

$$
\begin{aligned}
I_{\mathcal{X}_{\lambda}}= & (y, z) \cap(x, z) \cap(x+y-\lambda t, z) \cap \\
& (x, y, z)^{2} \cap(x-\lambda t, y, z)^{2} \cap(y-\lambda t, x, z)^{2} \\
= & \left(x^{2} y+x y^{2}-\lambda t x y, z\right) \cap \\
& (x, y, z)^{2} \cap(x-\lambda t, y, z)^{2} \cap(y-\lambda t, x, z)^{2} .
\end{aligned}
$$

A straightforward computation yields

$$
\begin{aligned}
& \qquad(x, y, z)^{2} \cap(x-\lambda t, y, z)^{2} \cap(y-\lambda t, x, z)^{2}= \\
& \left(x^{2} y^{2}, x y z, x^{2} z-\lambda t x z, y^{2} z-\lambda t y z, x^{2} y+x y^{2}-\lambda t x y, x^{4}-\lambda t x^{3}+\lambda^{2} t^{2} x^{2}, y^{4}-\lambda t y^{3}+\lambda^{2} t^{2} y^{2}, z^{2}\right) \\
& \text { and from here we get } \\
& \begin{aligned}
I_{\mathcal{X}_{\lambda}}= & \left(x^{2} y+x y^{2}-\lambda t x y\right)+ \\
& {\left[(z) \cap\left(x^{2} y^{2}, x y z, x^{2} z-\lambda t x z, y^{2} z-\lambda t y z, x^{4}-\lambda t x^{3}+\lambda^{2} t^{2} x^{2}, y^{4}-\lambda t y^{3}+\lambda^{2} t^{2} y^{2}, z^{2}\right)\right] } \\
= & \left(x^{2} y+x y^{2}-\lambda t x y\right)+ \\
& \left(x y z, x^{2} z-\lambda t x z, y^{2} z-\lambda t y z, x^{4} z-\lambda t x^{3} z+\lambda^{2} t^{2} x^{2} z, y^{4} z-\lambda t y^{3} z+\lambda^{2} t^{2} y^{2} z, z^{2}\right) .
\end{aligned}
\end{aligned}
$$

From this, setting $\lambda=0$, we obtain the ideal of $\mathcal{X}_{0}$, which is

$$
I_{\mathcal{X}_{0}}=\left(x^{2} y+x y^{2}, x y z, x^{2} z, y^{2} z, z^{2}\right) .
$$

On the other hand the scheme $L_{1}+L_{2}+L_{3,0}+\mathfrak{D}_{H, 3}(P)$, recalling that $\mathfrak{D}_{H, 3}(P)=$ $3 P \cap 2 H$, is defined by the ideal

$$
\begin{aligned}
I_{L_{1}} \cap I_{L_{2}} \cap I_{L_{3,0}} \cap\left[I_{P}^{3}+I_{H}^{2}\right] & =(y, z) \cap(x, z) \cap(x+y, z) \cap\left[(x, y, z)^{3}+\left(z^{2}\right)\right] \\
& =\left(x^{2} y+x y^{2}, z\right) \cap\left(x^{3}, y^{3}, x^{2} y, x y^{2}, x^{2} z, y^{2} z, x y z, z^{2}\right) \\
& =\left(x^{2} y+x y^{2}, x^{2} z, y^{2} z, x y z, z^{2}\right),
\end{aligned}
$$

which is exactly $I_{\mathcal{X}_{0}}$. Thus we see that the limiting scheme $\mathcal{X}_{0}$ is the $(2,3)$ cone configuration $L_{1}+L_{2}+L_{3,0}+\mathfrak{D}_{H, 3}(P)$, as we desired (see Figure 1). 

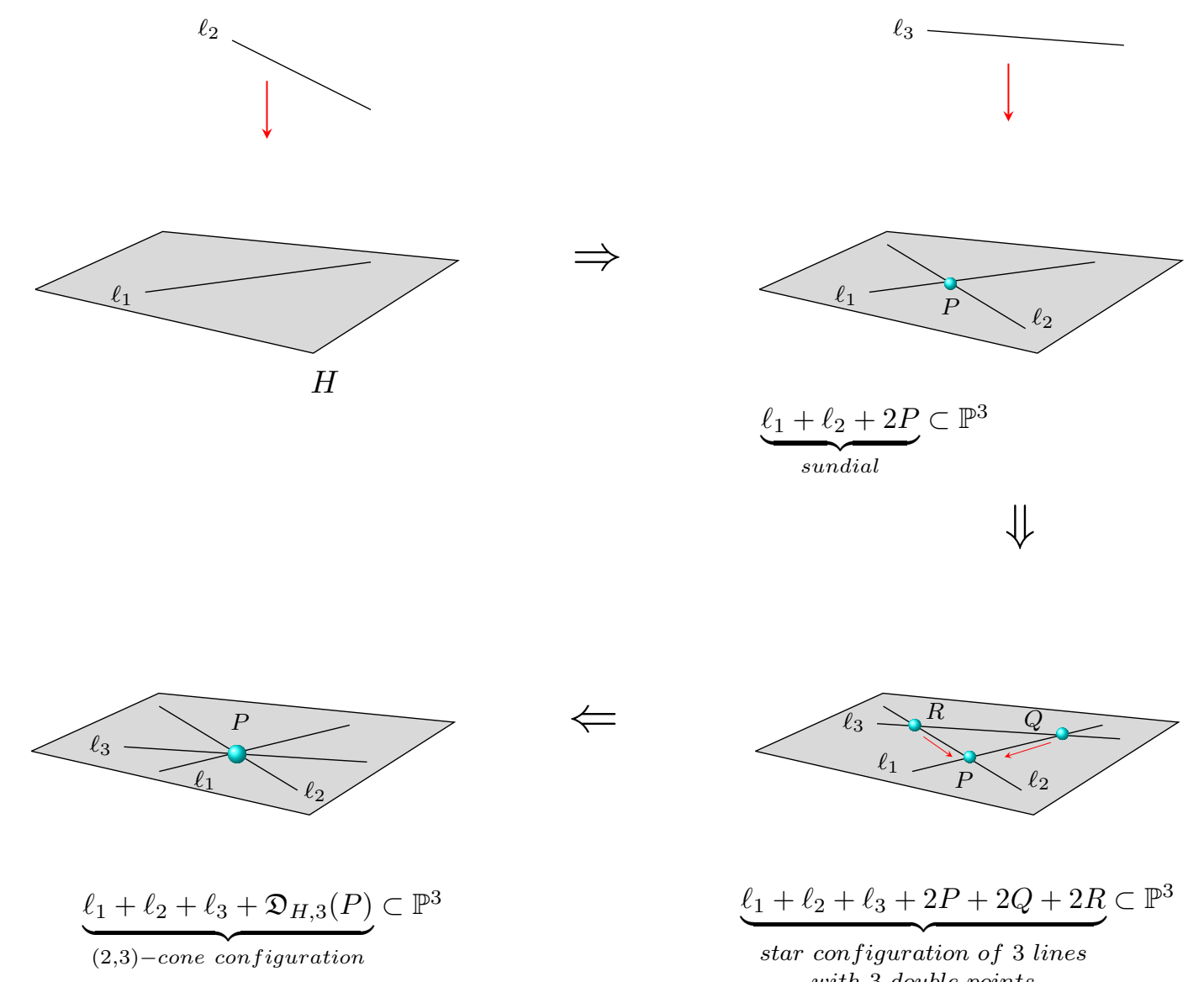

$$
\underbrace{\ell_{1}+\ell_{2}+2 P}_{\text {sundial }} \subset \mathbb{P}^{3}
$$

$\Downarrow$

Figure 1. The degeneration process to get a $(2,3)$-cone configuration

Summing up, this example illustrates that one can degenerate 3 disjoint lines in such a way that the resulting scheme becomes a $(2,3)$-cone configuration. Actually, we first degenerate 3 disjoint lines to a scheme of type $\mathcal{X}_{1}=L_{1}+$ $L_{2}+L_{3}+2 P+2 Q+2 R$, next we construct a flat family $\left\{\mathcal{X}_{\lambda}\right\}_{\lambda \in k}$ in such a way that whose general fiber $\mathcal{X}_{\lambda}=L_{1}+L_{2}+L_{3, \lambda}+2 P+2 Q_{\lambda}+2 R_{\lambda}$ approaches the $(2,3)$-cone configuration $\mathcal{X}_{0}=L_{1}+L_{2}+L_{3,0}+\mathfrak{D}_{H, 3}(P)$ when $\lambda \rightarrow 0$. This means that a $(2,3)$-cone configuration can be viewed as a degeneration of a star configuration of 3 lines with 3 double points, consequently, as a degeneration of 3 disjoint lines.

In addition, we easily find that

$$
\begin{aligned}
I_{\mathcal{X}_{0}}:(z) & =\left(x^{2} y+x y^{2}, x y z, x^{2} z, y^{2} z, z^{2}\right):(z) \\
& =\left(x^{2}, y^{2}, x y, z\right)
\end{aligned}
$$


that is $\left[(x, y, z)^{2}+(z)\right]$, i.e. the ideal of $\left.2 P\right|_{H}$, hence

$$
\operatorname{Res}_{H}\left(\mathcal{X}_{0}\right)=\left.2 P\right|_{H}
$$

Also,

$$
\begin{aligned}
I_{\mathcal{X}_{0}}+(z) & =\left(x^{2} y+x y^{2}, x y z, x^{2} z, y^{2} z, z^{2}\right)+(z) \\
& =\left(x^{2} y+x y^{2}, z\right),
\end{aligned}
$$

that is $[(y, z) \cap(x, z) \cap(x+y, z)]$, i.e. the ideal of $L_{1}+L_{2}+L_{3,0}$, so

$$
\operatorname{Tr}_{H}\left(\mathcal{X}_{0}\right)=L_{1}+L_{2}+L_{3,0} .
$$

\subsection{A Degeneration Inductive Method: How $(2, \mathbf{s})$-Cone Configura-} tions Appear as Degenerations of $s$ Skew Lines? One can generalize the result of $\S 3.2$, which dealt with the degeneration of 3 disjoint lines exclusively, so as to also deal with the degeneration of $s$ disjoint lines, provided that a disjoint union of $s$ lines can be degenerated to a $(2, s)$-cone configuration. In fact our method appearing in $§ 3.2$ can be successfully extended to the general case. Suppose that $L_{1}, \ldots, L_{s}$ be $s$ disjoint lines in $\mathbb{P}^{3}$ and that $H$ be a fixed plane containing $L_{1}$. (Note that by abuse of notation if we specialize some line $L_{i}$, we will again denote it by $L_{i}$ in the sequel). To begin, we specialize the line $L_{2}$ into $H$, so that we have the sundial $L_{1}+L_{2}+2 P$. Next we proceed in $s-2$ steps by an inductive argument based on adding one line at each inductive step, specializing it to lie on $H$, and finally degenerating it in an effective way for the purpose of getting desired cone configuration. Indeed, starting with the sundial $L_{1}+L_{2}+2 P$, we can lift step by step, to get the $(2, i)$-cone configuration $L_{1}+\cdots+L_{i}+\mathfrak{D}_{H, i}(P)$, for each $i, 3 \leq i \leq s$. Let us explain the process in details. The initial step is exactly the same as $§ 3.2$, which consists of specializing the line $L_{3}$ into $H$ that meets $L_{1}, L_{2}$ in 2 distinct points $P_{1,3}=L_{1} \cap L_{3}, P_{2,3}=L_{2} \cap L_{3}$, and moving $L_{3}$ so that $P_{1,3}, P_{2,3}$ approach the point $P$. Hence what we get in this step is the $(2,3)$-cone configuration $L_{1}+L_{2}+L_{3}+\mathfrak{D}_{H, 3}(P)$, as described in $\S 3.2$. In the second step, first specialize the line $L_{4}$ into $H$ so that this line meets each of the previous lines $L_{1}, L_{2}, L_{3}$ in distinct points $P_{1,4}=L_{1} \cap L_{4}, P_{2,4}=L_{2} \cap L_{4}$ and $P_{3,4}=L_{3} \cap L_{4}$, where, in view of Lemma 3.2, the specialized scheme is obviously

$$
\left[L_{1}+L_{2}+L_{3}+\mathfrak{D}_{H, 3}(P)\right]+\left[L_{4}+2 P_{1,4}+2 P_{2,4}+2 P_{3,4}\right] .
$$

Then let the line $L_{4}$ move in such a way that the points $P_{1,4}, P_{2,4}, P_{3,4}$ approach the point $P$, so, using the same reasoning as the previous step (for which we refer to Lemma 3.7), we get the $(2,4)$-cone configuration $L_{1}+L_{2}+L_{3}+L_{4}+$ $\mathfrak{D}_{H, 4}(P)$. Continuing in this way, we arrive at step $\mathbf{i}, 1 \leq i \leq s-2$, which can be performed as follows: 
- specialize the line $L_{i+2}$ into $H$ so that this line meets each of the previous lines $L_{1}, \ldots, L_{i+1}$ in distinct points

$$
P_{1, i+2}=L_{1} \cap L_{i+2} ; \ldots ; P_{i+1, i+2}=L_{i+1} \cap L_{i+2} ;
$$

- observe that, in view of Lemma 3.2, the outcome of this specialization is

$$
\left[L_{1}+\cdots+L_{i+1}+\mathfrak{D}_{H, i+1}(P)\right]+\left[L_{i+2}+2 P_{1, i+2}+\cdots+2 P_{i+1, i+2}\right] ;
$$

- move the specialized line $L_{i+2}$ in such a way that the $(i+1)$ points $P_{1, i+2}, \ldots, P_{i+1, i+2}$ approach the point $P$;

- find that the resulting scheme of this degeneration is the $(2, i+2)$-cone configuration

$$
L_{1}+\cdots+L_{i+2}+\mathfrak{D}_{H, i+2}(P) .
$$

The appealing fact of the inductive procedure is that the way one checks the resulting scheme in all the steps is the same (see Lemma 3.7 for a detailed proof). Eventually we see that in the last step the resulting degenerated scheme is the $(2, s)$-cone configuration

$$
L_{1}+\cdots+L_{s}+\mathfrak{D}_{H, s}(P)
$$

that is what we expected.

The above discussion leads to the following substantial improvement of the conclusion of $\S 3.2$, which asserts that a $(2, s)$-cone configuration is a flat limit of a flat family whose general fiber is the disjoint union of $s$ lines.

Lemma 3.7. Let $\mathcal{X} \subset \mathbb{P}^{3}$ be the disjoint union of $s$ lines $L_{1}, \ldots, L_{s}$, and $s \geq 3$. Then there exists a flat family of subschemes $\mathcal{X}_{\lambda} \subset \mathbb{P}^{3},(\lambda \in k)$, whose general fiber is the union of $s$ disjoint lines and whose special fiber is the $(2, s)$-cone configuration $\mathcal{X}_{0}=L_{1}+L_{2}^{\prime}+\cdots+L_{s}^{\prime}+\mathfrak{D}_{H, s}(P)$, where $H \cong \mathbb{P}^{2}$ is a generic plane containing the line $L_{1}$, and $L_{2}^{\prime}, \ldots, L_{s}^{\prime}$ are lines in $H$ passing through a point $P$, such that $L_{1} \cap L_{2}^{\prime} \cap \ldots \cap L_{s}^{\prime}=P$.

Proof. We proceed by induction on $s$. For $s=3$, the result is immediate from $\S 3.2$.

Now suppose that $s \geq 4$. Assume by induction that the lemma holds for $s-1$, which says that one can degenerate a disjoint union of $s-1$ lines to a $(2, s-1)$-cone configuration.

We can prove this lemma almost in the same way as in $\S 3.2$. However, some modifications will be need. We will not attempt to do this exhaustively, but will only indicate certain aspects of this general situation, and will mention what changes one needs to make.

Let $L_{1}, \ldots, L_{s}$ be $s$ disjoint lines in $\mathbb{P}^{3}$. First, by the induction hypothesis, one degenerates the $(s-1)$ lines $L_{1}, \ldots, L_{s-1}$ to the $(2, s-1)$-cone configuration 
$L_{1}+L_{2}^{\prime}+\cdots+L_{s-1}^{\prime}+\mathfrak{D}_{H, s-1}(P)$, where $H$ is a fixed plane containing the line $L_{1}$ and $P$ is the single intersection point of the lines $L_{1}, L_{2}^{\prime}, \ldots, L_{s-1}^{\prime}$. Considering the coordinates $t, x, y, z$ on $\mathbb{P}^{3}$, one may suppose that $P=[1: 0: 0: 0]$, and $H$ is the plane defined by the equation $z=0$, moreover the lines $L_{1}, L_{2}^{\prime}, \ldots, L_{s-1}^{\prime}$ are defined by the ideals

$$
\left\{\begin{array}{l}
I_{L_{1}}=(x, z) \\
I_{L_{2}^{\prime}}=(y, z) \\
I_{L_{3}^{\prime}}=(x+y, z) \\
I_{L_{4}^{\prime}}=(x+2 y, z) \\
\vdots \\
I_{L_{s-1}^{\prime}}=(x+(s-3) y, z)
\end{array}\right.
$$

(note that we can always degenerate the lines so that this is the case).

Next, specialize the line $L_{s}$ into $H$ such that this line meets each of the previous lines $L_{1}, L_{2}^{\prime}, \ldots, L_{s-1}^{\prime}$ in the following distinct points

$$
P_{1}=L_{1} \cap L_{s} ; P_{2}=L_{2}^{\prime} \cap L_{s} ; P_{3}=L_{3}^{\prime} \cap L_{s} ; \ldots ; P_{s-1}=L_{s-1}^{\prime} \cap L_{s} .
$$

Notice that we have the double structure at these intersection points, (see Lemma 3.2), so this implies that the specialized scheme becomes

$$
\left[L_{1}+L_{2}^{\prime}+\cdots+L_{s-1}^{\prime}+\mathfrak{D}_{H, s-1}(P)\right]+\left[L_{s}+2 P_{1}+\cdots+2 P_{s-1}\right],
$$

which we call $\mathcal{X}_{1}$.

We may assume that the line $L_{s}$ is defined by

$$
I_{L_{s}}=(x-y-t, z) \text {. }
$$

hence we have

$$
\left\{\begin{array}{l}
P_{1}=[1: 0:-1: 0] \\
P_{2}=[1: 1: 0: 0] \\
P_{3}=[1: 1 / 2:-1 / 2: 0] ; \\
P_{4}=[1: 2 / 3:-1 / 3: 0] \\
\vdots \\
P_{s-1}=[1:(s-3) /(s-2):-1 /(s-2): 0] .
\end{array}\right.
$$

Now let the line $L_{s}$ move in such a way that the $(s-1)$ points $P_{1}, \ldots, P_{s-1}$ approach the point $P=[1: 0: 0: 0]$. In order to find the resulting scheme of this degeneration, consider the flat family $\left\{\mathcal{X}_{\lambda}\right\}_{\lambda \in k}$, whereas $\mathcal{X}_{\lambda}$ is the scheme

$$
\left[L_{1}+L_{2}^{\prime}+\cdots+L_{s-1}^{\prime}+\mathfrak{D}_{H, s-1}(P)\right]+\left[L_{s, \lambda}+2 P_{1, \lambda}+\cdots+2 P_{s-1, \lambda}\right],
$$


where the points $P_{1, \lambda}, \ldots, P_{s-1, \lambda}$,

$$
\left\{\begin{array}{l}
P_{1, \lambda}=[1: 0:-\lambda: 0] \\
P_{2, \lambda}=[1: \lambda: 0: 0] \\
P_{3, \lambda}=[1: \lambda / 2:-\lambda / 2: 0] ; \\
P_{4, \lambda}=[1: 2 \lambda / 3:-\lambda / 3: 0] ; \\
\vdots \\
P_{s-1, \lambda}=[1: \lambda(s-3) /(s-2):-\lambda /(s-2): 0],
\end{array}\right.
$$

move to the point $P$, as well as the line joining $L_{s, \lambda}$, when $\lambda$ tends to 0 . That is equivalent to setting that

$$
I_{L_{s, \lambda}}=(x-y-\lambda t, z)
$$

and

$$
\left\{\begin{array}{l}
I_{P_{1, \lambda}}=(x, x-y-\lambda t, z) \\
I_{P_{2, \lambda}}=(y, x-y-\lambda t, z) \\
I_{P_{3, \lambda}}=(x+y, x-y-\lambda t, z) \\
I_{P_{4, \lambda}}=(x+2 y, x-y-\lambda t, z) \\
\vdots \\
I_{P_{s-1, \lambda}}=(x+(s-3) y, x-y-\lambda t, z)
\end{array}\right.
$$

From (4), observe that

$$
\begin{gathered}
I_{\mathcal{X}_{\lambda}}=\left[I_{L_{1}} \cap I_{L_{2}^{\prime}} \cap \ldots \cap I_{L_{s-1}^{\prime}} \cap\left(I_{P}^{s-1}+I_{H}^{2}\right)\right] \\
\cap\left[I_{L_{s, \lambda}} \cap I_{P_{1, \lambda}}^{2} \cap \ldots \cap I_{P_{s-1, \lambda}}^{2}\right] .
\end{gathered}
$$

Now to get the limiting scheme $\mathcal{X}_{0}$, we continue as in $\S 3.2$, but by using a longer and more tedious calculation, that we have not performed here. Indeed, by substituting (3), (5), (6) in the right hand side of the equality (7); then calculating in a similar but more complicated way to that carried out for $\S 3.2$ (which can be done by using $\mathrm{CoCoA}[\mathrm{CoC} 04]$ ); and finally letting $\lambda=0$; we obtain that

$$
I_{\mathcal{X}_{0}}=I_{L_{1}} \cap I_{L_{2}^{\prime}} \cap \ldots \cap I_{L_{s-1}^{\prime}} \cap I_{L_{s, 0}} \cap\left(I_{P}^{s}+I_{H}^{2}\right),
$$

that is, denoting by $L_{s}^{\prime}$ the line $L_{s, 0}$, the ideal of the $(2, s)$-cone configuration

$$
L_{1}+L_{2}^{\prime}+\cdots+L_{s-1}^{\prime}+L_{s}^{\prime}+\mathfrak{D}_{H, s}(P),
$$

as expected.

Although the above proof is a very natural way to find the limiting scheme $\mathcal{X}_{0}$, we would like to provide a different proof by finding the limits of residual and trace of the scheme $\mathcal{X}_{\lambda}$ with respect to the plane $H$. In fact to prove that

$$
\mathcal{X}_{0}=L_{1}+L_{2}^{\prime}+\cdots+L_{s-1}^{\prime}+L_{s}^{\prime}+\mathfrak{D}_{H, s}(P),
$$


it suffices, by construction, to prove that

$$
\operatorname{Tr}_{H}\left(\mathcal{X}_{0}\right)=L_{1}+L_{2}^{\prime}+\cdots+L_{s-1}^{\prime}+L_{s}^{\prime}
$$

and

$$
\operatorname{Res}_{H}\left(\mathcal{X}_{0}\right)=\mathfrak{D}_{H, s-1}(P),
$$

which we will verify as follows.

With regard to trace scheme $\operatorname{Tr}_{H}\left(\mathcal{X}_{\lambda}\right)$, using (7), (3), (5), (6) and noting that $I_{P}=(x, y, z), I_{H}=(z)$, we have

$$
\begin{aligned}
I_{\mathcal{X}_{\lambda}}+(z)= & \left\{(x, z) \cap(y, z) \cap(x+y, z) \cap \ldots \cap(x+(s-3) y, z) \cap\left[(x, y, z)^{s-1}+\left(z^{2}\right)\right]\right. \\
& \cap(x-y-\lambda t, z) \cap(x, x-y-\lambda t, z)^{2} \cap(y, x-y-\lambda t, z)^{2} \\
& \left.\cap(x+y, x-y-\lambda t, z)^{2} \cap \ldots \cap(x+(s-3) y, x-y-\lambda t, z)^{2}\right\}+(z) \\
= & \left\{(x) \cap(y) \cap(x+y) \cap \ldots \cap(x+(s-3) y) \cap(x, y)^{s-1}\right. \\
& \cap(x-y-\lambda t) \cap(x, x-y-\lambda t)^{2} \cap(y, x-y-\lambda t)^{2} \\
& \left.\cap(x+y, x-y-\lambda t)^{2} \cap \ldots \cap(x+(s-3) y, x-y-\lambda t)^{2}\right\}+(z) \\
= & (x y(x+y) \cdots(x+(s-3) y)(x-y-\lambda t))+(z),
\end{aligned}
$$

and from here by setting $\lambda=0$, we obtain the ideal of $\operatorname{Tr}_{H}\left(\mathcal{X}_{0}\right)$, that is

$$
\begin{aligned}
I_{\mathcal{X}_{0}}+(z) & =(x y(x+y) \cdots(x+(s-3) y)(x-y))+(z) \\
& =I_{L_{1}} \cap I_{L_{2}^{\prime}} \cap I_{L_{3}^{\prime}} \cap \ldots \cap I_{L_{s-1}^{\prime}} \cap I_{L_{s, 0}}
\end{aligned}
$$

so we get

$$
\operatorname{Tr}_{H}\left(\mathcal{X}_{0}\right)=L_{1}+L_{2}^{\prime}+L_{3}^{\prime}+\cdots+L_{s-1}^{\prime}+L_{s, 0} .
$$

On the other hand with regard to residual scheme $\operatorname{Res}_{H}\left(\mathcal{X}_{\lambda}\right)$, using (7), (3), (5), (6) and noting that $I_{P}=(x, y, z), I_{H}=(z)$, we have

$$
\begin{aligned}
I_{\mathcal{X}_{\lambda}}:(z)= & \left\{(x, z) \cap(y, z) \cap(x+y, z) \cap \ldots \cap(x+(s-3) y, z) \cap\left[(x, y, z)^{s-1}+\left(z^{2}\right)\right]\right. \\
& \cap(x-y-\lambda t, z) \cap(x, x-y-\lambda t, z)^{2} \cap(y, x-y-\lambda t, z)^{2} \\
& \left.\cap(x+y, x-y-\lambda t, z)^{2} \cap \ldots \cap(x+(s-3) y, x-y-\lambda t, z)^{2}\right\}:(z) \\
= & \left\{\left[(x, y, z)^{s-1}+\left(z^{2}\right)\right]:(z)\right\} \\
& \cap\left[(x, x-y-\lambda t, z)^{2}:(z)\right] \cap\left[(y, x-y-\lambda t, z)^{2}:(z)\right] \\
& \cap\left[(x+y, x-y-\lambda t, z)^{2}:(z)\right] \cap \ldots \cap\left[(x+(s-3) y, x-y-\lambda t, z)^{2}:(z)\right] \\
= & {\left[(x, y, z)^{s-2}+(z)\right] \cap(x, x-y-\lambda t, z) \cap(y, x-y-\lambda t, z) } \\
& \cap(x+y, x-y-\lambda t, z) \cap \ldots \cap(x+(s-3) y, x-y-\lambda t, z) \\
= & {\left[(x, y)^{s-2}+(z)\right] \cap(x y(x+y) \cdots(x+(s-3) y), x-y-\lambda t, z) } \\
= & \left\{(x, y)^{s-2} \cap(x y(x+y) \cdots(x+(s-3) y), x-y-\lambda t)\right\}+(z) \\
= & (x y(x+y) \cdots(x+(s-3) y))+(x, y)^{s-2}(x-y-\lambda t)+(z),
\end{aligned}
$$


and from here by letting $\lambda=0$, we obtain the ideal of $\operatorname{Res}_{H}\left(\mathcal{X}_{0}\right)$, that is

$$
\begin{aligned}
I_{\mathcal{X}_{0}}:(z) & =(x y(x+y) \cdots(x+(s-3) y))+(x, y)^{s-2}(x-y)+(z) \\
& =(x, y)^{s-1}+(z) \\
& =(x, y, z)^{s-1}+(z),
\end{aligned}
$$

so we have

$$
\operatorname{Res}_{H}\left(\mathcal{X}_{0}\right)=\left.(s-1) P\right|_{H}=\mathfrak{D}_{H, s-1}(P) .
$$

Now putting together (8) and (9), and denoting by $L_{s}^{\prime}$ the line $L_{s, 0}$, yields that

$$
\mathcal{X}_{0}=L_{1}+L_{2}^{\prime}+\cdots+L_{s-1}^{\prime}+L_{s}^{\prime}+\mathfrak{D}_{H, s}(P),
$$

which is the $(2, s)$-cone configuration as we wanted.

By what we have seen, the proof involves two kinds of degenerations. The first one is the degeneration inductive process obtained by successive degeneration techniques as explained above, which degenerates a disjoint union $\mathcal{X}$ of $s$ lines to the scheme of type $\mathcal{X}_{1}$. The second one is the degeneration of the scheme $\mathcal{X}_{1}$ to the $(2, s)$-cone configuration $\mathcal{X}_{0}$, where the main effort of the proof goes toward in. These degenerations can be combined in order to give rise to the degeneration of $\mathcal{X}$ to $\mathcal{X}_{0}$, and that is what we wanted to show.

Remark 3.8. A $(2, s)$-cone configuration as a degeneration of $s$ disjoint lines can be obtained in a slightly different way. In fact, one can degenerate $s$ disjoint lines $L_{1}, \ldots, L_{s}$ to a star configuration in a fixed plane $H$ containing $L_{1}$, and denote them again by the same letters as before. Call $P$ the intersection point of $L_{1}$ with $L_{2}$. Then let the lines $L_{3}, \ldots, L_{s}$, perhaps one by one, move in such a way that all the points of this star configuration unless the point $P$, which are $\left(\begin{array}{l}s \\ 2\end{array}\right)-1$ points, approach $P$. Now one finds that the resulting degenerated scheme is nothing but the desired $(2, s)$-cone configuration

$$
L_{1}+\cdots+L_{s}+\mathfrak{D}_{H, s}(P),
$$

(see Figure 1 for $(2,3)$-cone configuration).

At the end it is worth mentioning that although in the present paper we only deal with the case of $(2, s)$-cone configuration as a degeneration of $s$ disjoint lines in $\mathbb{P}^{3}$, one might be tempted to generalize Lemma 3.7 to higher dimensional ambient spaces $\mathbb{P}^{n}$. In fact, to be generally applicable in the induction argument discussed in the this section, the strong requirement that the degeneration process takes place in a flat family is needed. As the following lemma states, this is certainly feasible.

Lemma 3.9. There exists a flat family $\left\{\mathcal{X}_{\lambda}\right\}_{\lambda \in k}$ of subschemes in $\mathbb{P}^{n}$, with $s, n \geq 3$, whose general fiber is a disjoint union of $s$ lines and whose special fiber is a $(2, s)$-cone configuration. 
Proof. The proof follows an immediate generalization of the argument used in the proof of Lemma 3.7.

\section{Reduction Process}

We are now ready to prove the main theorem of this paper (Theorem 1.1 in Introduction):

Theorem 4.1. Let $X \subset \mathbb{P}^{3}$ be a generic union of e lines. Then $X$ has good postulation, i.e.,

$$
h^{0}\left(\mathcal{I}_{X}(d)\right)=\max \left\{\left(\begin{array}{c}
d+3 \\
3
\end{array}\right)-e(d+1), 0\right\} .
$$

In this section, we explain a reformulation of Theorem 4.1, that is the good postulation statement $\mathbf{H}_{d}$; next, we describe the inductive procedure involving slightly different good postulation statements in lower degrees (i.e. $\mathbf{H}_{d-1}^{\prime}$ ) and lower dimensions (i.e. $\mathbf{H}_{d}^{\prime \prime}$ ) which we will use to prove $\mathbf{H}_{d}$.

4.1. Auxiliary Vanishing Statements. Let us illustrate our method for proving Theorem 4.1.

In the setting of the theorem, as $X$ varies in a flat family, by the semicontinuity of cohomology [Har77, III, 12.8], the condition of good postulation, is clearly an open condition on the family of $X$. Hence to prove Theorem 4.1 , it is enough to find any scheme of $e$ lines, or even any scheme which is a specialization of a flat family of $e$ lines, which has good postulation.

For each $d \in \mathbb{N}$ consider the statement:

$\left(\mathbf{H}_{d}\right)$ : Assume that

$$
r=\left\lfloor\frac{\left(\begin{array}{c}
d+3 \\
3
\end{array}\right)}{d+1}\right\rfloor ; \quad q=\left(\begin{array}{c}
d+3 \\
3
\end{array}\right)-r(d+1) .
$$

Let the scheme $X \subset \mathbb{P}^{3}$ be the generic union of $r$ lines $L_{1}, \ldots, L_{r}$, and $q$ points $P_{1}, \ldots, P_{q}$ lying on a generic line $M$. Then $X$ has good postulation, i.e.,

$$
h^{1}\left(\mathcal{I}_{X}(d)\right)=h^{0}\left(\mathcal{I}_{X}(d)\right)=\left(\begin{array}{c}
d+3 \\
3
\end{array}\right)-r(d+1)-q=0 .
$$

Using Remark 2.3, Theorem 4.1 follows immediately from the statement $\mathbf{H}_{d}$, as explained below. Given $d$, suppose that $X$ is the scheme as in the statement $\mathbf{H}_{d}$. To prove Theorem 4.1 for any $e<r$, if one removes the $q$ points and $r-e$ lines from $X$, one gets a scheme $Y \subset X$ consisting of $e$ disjoint lines, then $h^{1}\left(\mathcal{I}_{X}(d)\right)=0$ implies that $h^{1}\left(\mathcal{I}_{Y}(d)\right)=0$ (by Remark 2.3 (i)). To prove Theorem 4.1 for any $e>r$, if one adds the line $M$ passing through the $q$ collinear points, as well as adds $e-r-1$ disjoint lines, to $X$, 
one gets a scheme $Y \supset X$ consisting of $e$ disjoint lines, hence $h^{0}\left(\mathcal{I}_{X}(d)\right)=0$ gives $h^{0}\left(\mathcal{I}_{Y}(d)\right)=0$ (by Remark 2.3 (ii)).

Looking quickly at the base cases $d=1,2$ shows that the assertions $\mathbf{H}_{1}$ and $\mathbf{H}_{2}$ are quite trivial:

$\left(\mathbf{H}_{1}\right)$ : If $X$ is a union of 2 skew lines in $\mathbb{P}^{3}$, then $h^{0}\left(\mathcal{I}_{X}(1)\right)=0$, which means that there is no plane containing $X$; (this is so obvious).

$\left(\mathbf{H}_{2}\right)$ : If $X$ is a generic union of 3 lines and one point in $\mathbb{P}^{3}$, then $h^{0}\left(\mathcal{I}_{X}(2)\right)=$ 0 , which means that there is no quadric surface containing $X$; (this is clear, because of the fact that through 3 disjoint lines in $\mathbb{P}^{3}$ there passes a unique quadric surface, so it is suffices to take the point outside this quadric).

It remains thus just to verify $\mathbf{H}_{d}$ for the cases $d \geq 3$. In order to show this assertion in its generality, we need to consider two further statements for each $d \geq 3$, namely the following:

$\left(\mathbf{H}_{d-1}^{\prime}\right)$ : Taking $r$ as defined in (10), assume that

$$
m=\left\lfloor\frac{d}{3}\right\rfloor+1 ; \quad s=\left(\begin{array}{c}
m \\
2
\end{array}\right)+(r-m) d-\left(\begin{array}{c}
d+2 \\
3
\end{array}\right) ; \quad t=r-m-2 s
$$

Let $H$ be a fixed plane in $\mathbb{P}^{3}$, and let $P$ be a generic point on $H$. Let $L_{1}, \ldots, L_{t}$ be $t$ generic lines in $\mathbb{P}^{3}$, and let $C_{1}, \ldots, C_{s} \subset \mathbb{P}^{3}$ be $s$ generic degenerate conics with only their singular points on the plane $H$. If

$$
X^{\prime}=\left.(m-1) P\right|_{H}+L_{1}+\cdots+L_{t}+C_{1}+\cdots+C_{s} \subset \mathbb{P}^{3},
$$

then

$$
h^{0}\left(\mathcal{I}_{X^{\prime}}(d-1)\right)=0
$$

$\left(\mathbf{H}_{d}^{\prime \prime}\right): \quad$ Take $q$ as defined in (10), and take $m, s, t$ as defined in (11). Let the scheme $X^{\prime \prime} \subset \mathbb{P}^{2}$ be the generic union of one cone configuration of type $m$, $s$ double points, $t$ simple points, and $q$ points lying on a generic line. Then

$$
h^{0}\left(\mathcal{I}_{X^{\prime \prime}}(d)\right)=0 .
$$

Keep in mind the data $r, q, m, s, t$ as defined in (10) and (11) in the remainder of the paper. Note that a direct computation yields the following relations, which are extensively used in Sections 5 and 6, 
$(\ddagger)$ :

for $d \equiv 0(\bmod 3): \quad r=\frac{(d+2)(d+3)}{6} ; \quad q=0 ; \quad m=\frac{d+3}{3} ; \quad s=\left(\begin{array}{c}m-1 \\ 2\end{array}\right) ; \quad t=\left(\begin{array}{c}m+2 \\ 2\end{array}\right)-3$,

for $d \equiv 1(\bmod 3): \quad r=\frac{(d+2)(d+3)}{6} ; \quad q=0 ; \quad m=\frac{d+2}{3} ; \quad s=\left(\begin{array}{c}m \\ 2\end{array}\right) ; \quad t=\left(\begin{array}{c}m+1 \\ 2\end{array}\right)$,

for $d \equiv 2(\bmod 3): \quad r=\frac{(d+1)(d+4)}{6} ; \quad q=\frac{d+1}{3} ; \quad m=\frac{d+1}{3} ; \quad s=\left(\begin{array}{c}m \\ 2\end{array}\right) ; \quad t=\left(\begin{array}{c}m+2 \\ 2\end{array}\right)-1$.

4.2. $\mathbf{H}_{\mathbf{d}-\mathbf{1}}^{\prime}+\mathbf{H}_{\mathbf{d}}^{\prime \prime} \Rightarrow \mathbf{H}_{\mathbf{d}}$ for $\mathbf{d} \geq \mathbf{3}$. Our goal in this part will be to show that: if $d \geq 3$, then the statements $\mathbf{H}_{d-1}^{\prime}$ and $\mathbf{H}_{d}^{\prime \prime}$ imply $\mathbf{H}_{d}$. To begin, let the scheme $X$ as in the statement $\mathbf{H}_{d}$, where

$$
X=L_{1}+\cdots+L_{r}+P_{1}+\cdots+P_{q} \subset \mathbb{P}^{3},
$$

is the generic union of $r$ lines $L_{1}, \ldots, L_{r}$, and $q$ points $P_{1}, \ldots, P_{q}$ lying on the generic line $M$.

Fix a generic plane $H \cong \mathbb{P}^{2}$. For the purpose of getting $h^{0}\left(\mathcal{I}_{X}(d)\right)=0$, we wish to find a scheme $\widetilde{X}$ obtained from $X$ by different kind of specializations and degenerations in the most efficient way, in the sense that the desired vanishing $h^{0}\left(\mathcal{I}_{\tilde{X}}(d)\right)=0$ can be then achieved. Now, setting $m, s, t$ as defined in (11), we construct such $\tilde{X}$ from $X$ by specializing the $q$ collinear points into $H$, by degenerating $m$ lines to a $(2, m)$-cone configuration supported in $H$, and by degenerating $s$ other pairs of lines to sundials with the property that only their singular points contained in $H$. To be more precise:

- specialize the line $M$, consequently the points $P_{1}, \ldots, P_{q}$, into $H$, (by abuse of notation, we will again denote these specialized points by $\left.P_{1}, \ldots, P_{q}\right)$;

- degenerate $m$ of the lines $L_{i}$ to $(2, m)$-cone configuration

$$
\widehat{\mathfrak{C}}=\mathfrak{C}+\mathfrak{D}_{H, m}(P),
$$

where $\mathfrak{C}$ is a cone configuration of type $m$ in $H$, and $P$ is the singular point of $\mathfrak{C}$, (recall that $\mathfrak{D}_{H, m}(P)=m P \cap 2 H$ );

- degenerate the next $s$ pairs of lines $L_{i}$, so that they become $s$ sundials

$$
\widehat{C}_{i}=C_{i}+2 Q_{i} ; \quad(1 \leq i \leq s),
$$

where $C_{i}$ is a degenerate conic and $Q_{i}$ is the singular point of $C_{i}$; furthermore, specialize the points $Q_{i}$ into $H$;

- leave the remaining lines $L_{i}$, which are $t=r-m-2 s$ lines, generic outside $H$, denoted $L_{1}, \ldots, L_{t}$;

then let

$$
\widetilde{X}=\widehat{\mathfrak{C}}+\widehat{C}_{1}+\cdots+\widehat{C}_{s}+L_{1}+\cdots+L_{t}+P_{1}+\cdots+P_{q} \subset \mathbb{P}^{3} .
$$


We need to show that $h^{0}\left(\mathcal{I}_{\widetilde{X}}(d)\right)=0$, which consequently, by the semicontinuity of cohomology [Har77, III, 12.8], implies that $h^{0}\left(\mathcal{I}_{X}(d)\right)=0$. To do this, by Castelnuovo's inequality (Lemma 2.2), it would be enough to show that the following vanishings

$$
h^{0}\left(\mathcal{I}_{\operatorname{Res}_{H}(\widetilde{X})}(d-1)\right)=0 ; \quad h^{0}\left(H, \mathcal{I}_{\operatorname{Tr}_{H}(\widetilde{X})}(d)\right)=0 .
$$

First we consider the residual of $\widetilde{X}$ with respect to $H \cong \mathbb{P}^{2}$. By using the fact that $\operatorname{Res}_{H}(\widehat{\mathfrak{C}})=\left.(m-1) P\right|_{H}$, moreover by observing that $\operatorname{Res}_{H}\left(\widehat{C}_{i}\right)=C_{i}$, we get

$$
\operatorname{Res}_{H}(\tilde{X})=\left.(m-1) P\right|_{H}+C_{1}+\cdots+C_{s}+L_{1}+\cdots+L_{t} \subset \mathbb{P}^{3} .
$$

Note that $C_{1}, \ldots, C_{s}$ are $s$ generic degenerate conics in $\mathbb{P}^{3}$ with the property that only their singular points lie on the plane $H$. Then it is quite immediate to see that the scheme $\operatorname{Res}_{H}(\widetilde{X})$ is of the type $X^{\prime}$ as in the statement $\mathbf{H}_{d-1}^{\prime}$. So this implies that proving $h^{0}\left(\mathcal{I}_{\operatorname{Res}_{H}(\tilde{X})}(d-1)\right)=0$ is precisely what we have to verify for $\mathbf{H}_{d-1}^{\prime}$.

Now we consider the trace of $\widetilde{X}$ on the plane $H$. By letting the points $N_{i}=L_{i} \cap H,(1 \leq i \leq t)$, also by using the facts that $\operatorname{Tr}_{H}(\widehat{\mathfrak{C}})=\mathfrak{C}$ and that $\operatorname{Tr}_{H}\left(\widehat{C}_{i}\right)$ is the double point $\left.2 Q_{i}\right|_{H}$ in $H,(1 \leq i \leq s)$, we have

$\operatorname{Tr}_{H}(\tilde{X})=\mathfrak{C}+\left.2 Q_{1}\right|_{H}+\cdots+\left.2 Q_{s}\right|_{H}+N_{1}+\cdots+N_{t}+P_{1}+\cdots+P_{q} \subset H \cong \mathbb{P}^{2}$,

which is generic union in $H$ of the cone configuration $\mathfrak{C}$ of type $m, s$ double points, $t$ simple points, and $q$ collinear points. Hence it is straightforward to observe that the scheme $\operatorname{Tr}_{H}(\widetilde{X})$ is of the type $X^{\prime \prime}$ as in the statement $\mathbf{H}_{d}^{\prime \prime}$. It follows that proving $h^{0}\left(H, \mathcal{I}_{T_{H}(\tilde{X})}(d)\right)=0$ is exactly what we have to investigate for $\mathbf{H}_{d}^{\prime \prime}$.

\section{ProOF OF $\mathbf{H}_{d-1}^{\prime}$}

In this section we will prove the statement $\mathbf{H}_{d-1}^{\prime}$ for all $d \geq 3$, which for convenient we state again.

$$
\begin{aligned}
& \left(\mathbf{H}_{d-1}^{\prime}\right): \text { Let } d \geq 3 \text {, and } \\
& \qquad r=\left\lfloor\frac{\left(\begin{array}{c}
d+3 \\
3
\end{array}\right)}{d+1}\right\rfloor ; \quad m=\left\lfloor\frac{d}{3}\right\rfloor+1 ; \\
& s=\left(\begin{array}{c}
m \\
2
\end{array}\right)+(r-m) d-\left(\begin{array}{c}
d+2 \\
3
\end{array}\right) ; \quad t=r-m-2 s .
\end{aligned}
$$


Let $H$ be a fixed plane in $\mathbb{P}^{3}$, and $P$ be a generic point on $H$. Consider the scheme $X^{\prime}$ as

$$
X^{\prime}=\left.(m-1) P\right|_{H}+L_{1}+\cdots+L_{t}+C_{1}+\cdots+C_{s} \subset \mathbb{P}^{3},
$$

where $L_{1}, \ldots, L_{t}$ are $t$ generic lines in $\mathbb{P}^{3}$, and $C_{1}, \ldots, C_{s}$ are $s$ generic degenerate conics in $\mathbb{P}^{3}$ with the property that only their singular points, denoted $Q_{i}$ $(1 \leq i \leq s)$, lie on the plane $H$. Then

$$
h^{0}\left(\mathcal{I}_{X^{\prime}}(d-1)\right)=0 .
$$

Let us consider the initial case $d=3$. In this case we find that

$$
m=2 ; \quad s=0 ; \quad t=3,
$$

which means that

$$
X^{\prime}=P+L_{1}+L_{2}+L_{3} \subset \mathbb{P}^{3}
$$

where $P$ is a generic point on the plane $H$, and $L_{i}$ are three generic lines in $\mathbb{P}^{3}$ not lying in $H$. We specialize the line $L_{1}$ into $H$, and we again denote this specialized line by $L_{1}$, in addition we denote by $\widetilde{X^{\prime}}$ the scheme obtained from $X^{\prime}$ by this specialization. We have

$$
\operatorname{Res}_{H}\left(\widetilde{X^{\prime}}\right)=L_{2}+L_{3} \subset \mathbb{P}^{3},
$$

which is a union of two skew lines, so it is obvious that

$$
h^{0}\left(\mathcal{I}_{\operatorname{Res}_{H}\left(\widetilde{X^{\prime}}\right)}(1)\right)=0
$$

Also,

$$
\operatorname{Tr}_{H}\left(\widetilde{X^{\prime}}\right)=P+L_{1}+N_{2}+N_{3} \subset H \cong \mathbb{P}^{2}
$$

where $L_{i}$ meets $H$ in the point $N_{i},(i=2,3)$. Since the line $L_{1}$ is a fixed component for the curves of $H^{0}\left(H, \mathcal{I}_{\operatorname{Tr}_{H}\left(\widetilde{X^{\prime}}\right)}(2)\right)$, we get

$$
h^{0}\left(H, \mathcal{I}_{\operatorname{Tr}_{H}\left(\widetilde{X^{\prime}}\right)}(2)\right)=h^{0}\left(H, \mathcal{I}_{\operatorname{Tr}_{H}\left(\widetilde{X^{\prime}}\right)-L_{1}}(1)\right) .
$$

Observing that $\operatorname{Tr}_{H}\left(\widetilde{X^{\prime}}\right)-L_{1}$ is made by three generic points, it trivially follows

and from here

$$
h^{0}\left(H, \mathcal{I}_{\operatorname{Tr}_{H}\left(\widetilde{X^{\prime}}\right)-L_{1}}(1)\right)=0
$$

$$
h^{0}\left(H, \mathcal{I}_{\operatorname{Tr}_{H}\left(\widetilde{X^{\prime}}\right)}(2)\right)=0 .
$$

Putting together $h^{0}\left(\mathcal{I}_{\operatorname{Res}_{H}\left(\widetilde{X^{\prime}}\right)}(1)\right)=0$ and $h^{0}\left(H, \mathcal{I}_{\operatorname{Tr}_{H}\left(\widetilde{X^{\prime}}\right)}(2)\right)=0$, from Castelnuovo's inequality (Lemma 2.2) we get $h^{0}\left(\mathcal{I}_{\widetilde{X^{\prime}}}(2)\right)=0$, therefore the semicontinuity of cohomology [Har77, III, 12.8] implies that

$$
h^{0}\left(\mathcal{I}_{X^{\prime}}(2)\right)=0 \text {. }
$$

Hence the case $d=3$ is done, i.e. $\mathbf{H}_{2}^{\prime}$ is proved. 
Now assume $d \geq 4$. The rest of the proof will be by induction on $d$. We will investigate separately the three cases $d \equiv 0,1,2(\bmod 3)$.

Notation 5.1. To stress the numerical data $m, s, t$ for the scheme $X^{\prime}$ considered in the statement $\mathbf{H}_{d-1}^{\prime}$, we will sometimes use the notation $X_{(m, s, t ; d)}^{\prime}$.

5.1. Case $\mathbf{d} \equiv \mathbf{0}(\bmod 3)$. In this case we have, $($ see $(\ddagger))$,

$$
m=\frac{d+3}{3} ; \quad s=\left(\begin{array}{c}
m-1 \\
2
\end{array}\right) ; \quad t=\left(\begin{array}{c}
m+2 \\
2
\end{array}\right)-3 .
$$

In order to the task of proving $h^{0}\left(\mathcal{I}_{X^{\prime}}(d-1)\right)=0$, we make a specialization of $X^{\prime}$ via the plane $H$. Let $\widetilde{X^{\prime}}$ be the scheme obtained from $X^{\prime}$ by degenerating $m-1$ of the lines $L_{i}$ in such a way that they become a $(2, m-1)$-cone configuration $\widehat{\mathfrak{C}}$,

$$
\widehat{\mathfrak{C}}=\mathfrak{C}+\mathfrak{D}_{H, m-1}(R),
$$

where $\mathfrak{C}$ is a cone configuration of type $m-1$ in $H$ and $R$ is the singular point of $\mathfrak{C}$. The remaining lines $L_{i}$, which are $t^{\prime}=t-(m-1)=\left(\begin{array}{c}m+1 \\ 2\end{array}\right)-1$ lines, are generic not lying on $H$, and we denote them by $L_{1}, \ldots, L_{t^{\prime}}$. Then

$$
\widetilde{X^{\prime}}=\left.(m-1) P\right|_{H}+L_{1}+\cdots+L_{t^{\prime}}+\widehat{\mathfrak{C}}+C_{1}+\cdots+C_{s} \subset \mathbb{P}^{3} .
$$

Our goal is to show that $h^{0}\left(\mathcal{I}_{\widetilde{X^{\prime}}}(d-1)\right)=0$, which by the semicontinuity of cohomology [Har77, III, 12.8] gives the desired conclusion $h^{0}\left(\mathcal{I}_{X^{\prime}}(d-1)\right)=0$. Now to do so, by Castelnuovo's inequality (Lemma 2.2), our next task will be to show that

$$
h^{0}\left(\mathcal{I}_{\operatorname{Res}_{H}\left(\widetilde{X^{\prime}}\right)}(d-2)\right)=0 ; \quad h^{0}\left(H, \mathcal{I}_{\operatorname{Tr}_{H}\left(\widetilde{X^{\prime}}\right)}(d-1)\right)=0 .
$$

First we consider $\operatorname{Res}_{H}\left(\widetilde{X^{\prime}}\right)$. Since the scheme $\left.(m-1) P\right|_{H}$ is completely contained in the plane $H$, its residual with respect to $H$ is empty. Notice that

$$
\operatorname{Res}_{H}(\widehat{\mathfrak{C}})=\operatorname{Res}_{H}\left(\mathfrak{C}+\mathfrak{D}_{H, m-1}(R)\right)=\left.(m-2) R\right|_{H} .
$$

Letting $m^{\prime}=m-1=\frac{d}{3}$, we obtain

$$
\operatorname{Res}_{H}\left(\widetilde{X^{\prime}}\right)=L_{1}+\cdots+L_{t^{\prime}}+\left.\left(m^{\prime}-1\right) R\right|_{H}+C_{1}+\cdots+C_{s} \subset \mathbb{P}^{3},
$$

where $t^{\prime}=\left(\begin{array}{c}m^{\prime}+2 \\ 2\end{array}\right)-1$ and $s=\left(\begin{array}{c}m^{\prime} \\ 2\end{array}\right)$. So it is an immediate to see that $\operatorname{Res}_{H}\left(\widetilde{X^{\prime}}\right)$ is precisely of the type $X_{\left(m^{\prime}, s, t^{\prime} ; d-1\right)}^{\prime}$ as in $\mathbf{H}_{d-2}^{\prime}$ for the case of $d-1 \equiv 2(\bmod$ 3 ), (one can check this fact simply by substituting $d-1$ in line 3 of $(\ddagger)$ ). Then by induction hypothesis we have

$$
h^{0}\left(\mathcal{I}_{\operatorname{Res}_{H}\left(\widetilde{X^{\prime}}\right)}(d-2)\right)=0,
$$

so we are done with the residual scheme. 
Now we consider the trace scheme $\operatorname{Tr}_{H}\left(\widetilde{X^{\prime}}\right)$. Recalling that the singular point $Q_{i}$ of $C_{i}$ lies on the plane $H$, we conclude that the degenerate conic $C_{i}$ meets $H$ at $Q_{i}$ in degree 2, that is a 2-dot in $H$ with support at $Q_{i}$, which we denote by $Z_{i},(1 \leq i \leq s)$. Moreover $L_{i}$ meets $H$ at the simple point $N_{i}$, $\left(1 \leq i \leq t^{\prime}\right)$. So we get

$$
\operatorname{Tr}_{H}\left(\widetilde{X^{\prime}}\right)=\left.(m-1) P\right|_{H}+N_{1}+\cdots+N_{t^{\prime}}+\mathfrak{C}+Z_{1}+\cdots+Z_{s} \subset H \cong \mathbb{P}^{2} .
$$

We notice that $\mathfrak{C}$ is a cone configuration of type $m-1$, (i.e. a union of $m-1$ intersecting lines passing through a single point), thus $\mathfrak{C}$ is a fixed component for the curves of $H^{0}\left(H, \mathcal{I}_{\operatorname{Tr}_{H}\left(\widetilde{X^{\prime}}\right)}(d-1)\right)$. Removing the fixed component $\mathfrak{C}$ implies that

$$
h^{0}\left(H, \mathcal{I}_{\operatorname{Tr}_{H}\left(\widetilde{X^{\prime}}\right)}(d-1)\right)=h^{0}\left(H, \mathcal{I}_{\operatorname{Tr}_{H}\left(\widetilde{X^{\prime}}\right)-\mathfrak{C}}(d-m)\right),
$$

which leads us to compute $h^{0}\left(H, \mathcal{I}_{\operatorname{Tr}_{H}\left(\widetilde{X^{\prime}}\right)-\mathfrak{C}}(d-m)\right)$, (note that by definition $d-m \geq 1)$. By setting

$$
T=\left.(m-1) P\right|_{H}+Z_{1}+\cdots+Z_{s},
$$

we observe that $T$ is the generic union in $H$ of one $(m-1)$-multiple point and $s$-dots. Moreover, by using $d=3 m-3$ and $m \geq 2$, we see that $d-m \geq m-2$ always holds. Hence we can apply Lemma 2.6 to the scheme $T$ in degree $d-m$, and we then obtain

$$
\begin{aligned}
h^{0}\left(H, \mathcal{I}_{T}(d-m)\right) & =\max \left\{\left(\begin{array}{c}
d-m+2 \\
2
\end{array}\right)-\left(\begin{array}{c}
m \\
2
\end{array}\right)-2 s, 0\right\} \\
& =\max \left\{\left(\begin{array}{c}
2 m-1 \\
2
\end{array}\right)-\left(\begin{array}{c}
m \\
2
\end{array}\right)-2\left(\begin{array}{c}
m-1 \\
2
\end{array}\right), 0\right\} \\
& =\max \left\{\left(\begin{array}{c}
m+1 \\
2
\end{array}\right)-1,0\right\} \\
& =\left(\begin{array}{c}
m+1 \\
2
\end{array}\right)-1 .
\end{aligned}
$$

We have $\operatorname{Tr}_{H}\left(\widetilde{X^{\prime}}\right)-\mathfrak{C}=T+N_{1}+\cdots+N_{t^{\prime}}$, where the points $N_{1}, \ldots, N_{t^{\prime}}$ are $t^{\prime}=\left(\begin{array}{c}m+1 \\ 2\end{array}\right)-1$ generic points in $H$. So the equality

$$
h^{0}\left(H, \mathcal{I}_{T}(d-m)\right)=\left(\begin{array}{c}
m+1 \\
2
\end{array}\right)-1
$$

implies that

$$
h^{0}\left(H, \mathcal{I}_{\operatorname{Tr}_{H}\left(\widetilde{X^{\prime}}\right)-\mathfrak{C}^{\mathfrak{c}}}(d-m)\right)=0,
$$

which is, by (12), equivalent to

$$
h^{0}\left(H, \mathcal{I}_{\operatorname{Tr}_{H}\left(\widetilde{X^{\prime}}\right)}(d-1)\right)=0 .
$$


This finishes the proof.

5.2. Case $\mathbf{d} \equiv \mathbf{1}(\bmod 3)$. In this case we have, $($ see $(\ddagger))$,

$$
m=\frac{d+2}{3} ; \quad s=\left(\begin{array}{c}
m \\
2
\end{array}\right) ; \quad t=\left(\begin{array}{c}
m+1 \\
2
\end{array}\right) \text {. }
$$

Recall that the scheme

$$
X^{\prime}=\left.(m-1) P\right|_{H}+L_{1}+\cdots+L_{t}+C_{1}+\cdots+C_{s} \subset \mathbb{P}^{3},
$$

is generic union of the $t$ lines $L_{i}$, the $s$ degenerate conics $C_{i}$ with the property that only their singular points $Q_{i}$ lie on the plane $H$, and finally $\left.(m-1) P\right|_{H}$.

First consider the case $d=4$. We have $m=2, s=1, t=3$. (Note that we consider this case $d=4$ separately, because the general procedure requires that $s \geq m$ and $m \geq 3$, while for $d=4$ we have $s=1$ and $m=2$ ). Then

$$
X^{\prime}=P+L_{1}+L_{2}+L_{3}+C \subset \mathbb{P}^{3},
$$

where only the point $P$ and the singular point $Q$ of $C$ lie on $H$. We need to prove $h^{0}\left(\mathcal{I}_{X^{\prime}}(3)\right)=0$. Let $M_{1}, M_{2}$ be the two intersecting lines which form the degenerate conic $C$, that is $C=M_{1}+M_{2}$. Let $\widetilde{X^{\prime}}$ be the scheme obtained from $X^{\prime}$ by degenerating the lines $L_{1}$ and $M_{1}$ into $H$ so that they become a sundial $\widehat{C^{\prime}}=L_{1}^{\prime}+M_{1}^{\prime}+2 R$ with $R \neq Q$, where $\left(L_{1}^{\prime}+M_{1}^{\prime}\right)$ is a degenerate conic in $H$ and $2 R$ is double point in $\mathbb{P}^{3}$ with support at the singular point of $\left(L_{1}^{\prime}+M_{1}^{\prime}\right)$, (the lines $L_{2}, L_{3}, M_{2}$ remain generic not lying on $H$ ). Now by the semicontinuity of cohomology it suffices to prove $h^{0}\left(\mathcal{I}_{\widetilde{X}^{\prime}}(3)\right)=0$, whereas

$$
\widetilde{X^{\prime}}=P+L_{2}+L_{3}+M_{2}+\widehat{C^{\prime}} \subset \mathbb{P}^{3} .
$$

By having the fact that $\operatorname{Res}_{H}\left(\widehat{C^{\prime}}\right)=R$ and $\operatorname{Tr}_{H}\left(\widehat{C^{\prime}}\right)=L_{1}^{\prime}+M_{1}^{\prime}$, we easily get

$$
\begin{gathered}
\operatorname{Res}_{H} \widetilde{X^{\prime}}=L_{2}+L_{3}+M_{2}+R \subset \mathbb{P}^{3} ; \\
\operatorname{Tr}_{H} \widetilde{X^{\prime}}=P+N_{2}+N_{3}+L_{1}^{\prime}+M_{1}^{\prime} \subset H,
\end{gathered}
$$

where $N_{2}=L_{2} \cap H$ and $N_{3}=L_{3} \cap H$. The residual scheme $\operatorname{Res}_{H}\left(\widetilde{X^{\prime}}\right)$ is generic union of three lines and a simple point in $\mathbb{P}^{3}$, so it is clear (see $\left(\mathbf{H}_{2}\right)$ in $\S 4.1)$ that

$$
h^{0}\left(\mathcal{I}_{\operatorname{Res}_{H}\left(\widetilde{X^{\prime}}\right)}(2)\right)=0 .
$$

Moreover, note that every degree 3 curves lying on $H \cong \mathbb{P}^{2}$ and passing through $\operatorname{Tr}_{H} \widetilde{X^{\prime}}$ has to have the degenerate conic $\left(L_{1}^{\prime}+M_{1}^{\prime}\right)$ as a factor. Hence taking away this factor implies

$$
h^{0}\left(H, \mathcal{I}_{\operatorname{Tr}_{H}\left(\widetilde{X^{\prime}}\right)}(3)\right)=h^{0}\left(H, \mathcal{I}_{P+N_{2}+N_{3}}(1)\right),
$$


but the right hand side, where $P+N_{2}+N_{3}$ is generic union of three points in $H$, is trivially equal to zero. So we get the conclusion by putting the last equality together with (13), and then applying Castelnuovo's inequality.

From now on we assume that $d \geq 7$. This provides that $m \geq 3$ and $s \geq m$. To prove the vanishing $h^{0}\left(\mathcal{I}_{X^{\prime}}(d-1)\right)=0$, we introduce a scheme $\widetilde{X^{\prime}}$ obtained from $X^{\prime}$ by combining specializations and degenerations in the following way:

- Degenerate the lines $L_{1}, L_{2}$ to sundial

$$
\widehat{C}=C+2 Q,
$$

where $C$ is a degenerate conic and $Q$ is its singular point, furthermore, specialize only the point $Q$ into $H$.

- Consider the first $m$ degenerate conics $C_{i}$, and let $M_{1, i}, M_{2, i}$ be the two lines which form $C_{i},(1 \leq i \leq m)$, this means

$$
C_{i}=M_{1, i}+M_{2, i} ; \quad(1 \leq i \leq m) .
$$

Then degenerate $C_{1}, \ldots, C_{m}$ in such a way that the lines $M_{1,1}, \ldots, M_{1, m}$ become a $(2, m)$-cone configuration $\widehat{\mathfrak{C}}$ supported in $H$, that is

$$
\widehat{\mathfrak{C}}=\mathfrak{C}+\mathfrak{D}_{H, m}(R),
$$

where $\mathfrak{C}$ is a cone configuration of type $m$ in $H$ and $R$ is the singular point of $\mathfrak{C}$. Leave the lines $M_{2,1}, \ldots, M_{2, m}$ remain generic lines, not lying on $H$.

Now let

$\widetilde{X^{\prime}}=\left.(m-1) P\right|_{H}+\widehat{C}+L_{3}+\cdots+L_{t}+\widehat{\mathfrak{C}}+M_{2,1}+\cdots+M_{2, m}+C_{m+1}+\cdots+C_{s} \subset \mathbb{P}^{3}$.

We perform the process of verifying the residual and the trace of the scheme $\widetilde{X^{\prime}}$ with respect to the plane $H$, to get $h^{0}\left(\mathcal{I}_{\widetilde{X^{\prime}}}(d-1)\right)=0$, which automatically gives our required vanishing $h^{0}\left(\mathcal{I}_{X^{\prime}}(d-1)\right)=0$.

Let us consider the residual scheme. Since the scheme $\left.(m-1) P\right|_{H}$ is completely contained in the plane $H$, its residual with respect to $H$ is empty. Since the sundial $\widehat{C}=C+2 Q$ has only its singular point on $H$, we see that $\operatorname{Res}_{H}(\widehat{C})=C$. Moreover, by recalling that the cone configuration $\mathfrak{C}$ is contained in $H$ and that $\mathfrak{D}_{H, m}(R)=m R \cap 2 H$, we have

$$
\operatorname{Res}_{H}(\widehat{\mathfrak{C}})=\operatorname{Res}_{H}\left(\mathfrak{C}+\mathfrak{D}_{H, m}(R)\right)=\left.(m-1) R\right|_{H} .
$$

So we obtain

$\operatorname{Res}_{H}\left(\widetilde{X^{\prime}}\right)=C+L_{3}+\cdots+L_{t}+\left.(m-1) R\right|_{H}+M_{2,1}+\cdots+M_{2, m}+C_{m+1}+\cdots+C_{s} \subset \mathbb{P}^{3}$.

Observe that $\operatorname{Res}_{H}\left(\widetilde{X^{\prime}}\right)$ is generic union of the lines $L_{3}, \ldots, L_{t}, M_{2,1}, \ldots, M_{2, m}$, which are $t^{\prime}=(t-2)+m=\left(\begin{array}{c}m+1 \\ 2\end{array}\right)-2+m=\left(\begin{array}{c}m+2 \\ 2\end{array}\right)-3$ lines; and the degenerate conics $C, C_{m+1}, \ldots, C_{s}$, which are $s^{\prime}=1+(s-m)=1+\left(\begin{array}{c}m \\ 2\end{array}\right)-m=\left(\begin{array}{c}m-1 \\ 2\end{array}\right)$ 
degenerate conics (whose singular points lie on $H$ ); and finally $\left.(m-1) R\right|_{H}$. Then it is immediately clear that $\operatorname{Res}_{H}\left(\widetilde{X^{\prime}}\right)$ is of the type $X_{\left(m, s^{\prime}, t^{\prime} ; d-1\right)}^{\prime}$ as in $\mathbf{H}_{d-2}^{\prime}$ for the case of $d-1 \equiv 0(\bmod 3)$, (to check this it is enough to substitute $d-1$ in the first line of $(\ddagger))$. Thus by induction hypothesis we get

$$
h^{0}\left(\mathcal{I}_{\operatorname{Res}_{H}\left(\widetilde{X^{\prime}}\right)}(d-2)\right)=0 .
$$

Let us consider the trace scheme. On the one hand we have degenerated the lines $M_{1,1}, \ldots, M_{1, m}$ to the $(2, m)$-cone configuration $\widehat{\mathfrak{C}}$ supported in $H$, so $\operatorname{Tr}_{H}(\widehat{\mathfrak{C}})$ will be the cone configuration $\mathfrak{C}$ of type $m$ in $H$, which we denoted by $\mathfrak{C}=M_{1,1}^{\prime}+\cdots+M_{1, m}^{\prime} \subset H$. On the other hand the line $M_{2, i},(1 \leq i \leq m)$, meets $H$ in a point which is $M_{1, i}^{\prime} \cap M_{2, i}$, that is already contained in $M_{1, i}^{\prime}$ and then in $\mathfrak{C}$. So

$$
\operatorname{Tr}_{H}\left(\widehat{\mathfrak{C}}+M_{2,1}+\cdots+M_{2, m}\right)=\mathfrak{C}
$$

We then obtain

$\operatorname{Tr}_{H}\left(\widetilde{X^{\prime}}\right)=\left.(m-1) P\right|_{H}+\left.2 Q\right|_{H}+N_{3}+\cdots+N_{t}+\mathfrak{C}+Z_{m+1}+\cdots+Z_{s} \subset H \cong \mathbb{P}^{2}$, where $\left.2 Q\right|_{H}=\operatorname{Tr}_{H}(\widehat{C})$ is a double point in $H$ with support at $Q ; N_{i}=L_{i} \cap H$ is a simple point, $(3 \leq i \leq t)$; and lastly $Z_{i}=C_{i} \cap H$ is a 2-dot in $H$ with support at the point $Q_{i},(m+1 \leq i \leq s)$.

Here our goal is to prove $h^{0}\left(H, \mathcal{I}_{\operatorname{Tr}_{H}\left(\widetilde{X^{\prime}}\right)}(d-1)\right)=0$. According to the elementary observation that the cone configuration $\mathfrak{C}$ of type $m$ is a fixed component for the curves of $H^{0}\left(H, \mathcal{I}_{\operatorname{Tr}_{H}\left(\widetilde{X^{\prime}}\right)}(d-1)\right)$, this is equivalent to prove

$$
h^{0}\left(H, \mathcal{I}_{\operatorname{Tr}_{H}\left(\widetilde{X^{\prime}}\right)-\mathfrak{C}^{(}}(d-1-m)\right)=0 .
$$

Noting that $m=\frac{d+2}{3}$ and consequently $d-1-m=2 m-3$, we have to show that

whereas

$$
h^{0}\left(H, \mathcal{I}_{\operatorname{Tr}_{H}\left(\widetilde{X^{\prime}}\right)-\mathfrak{C}}(2 m-3)\right)=0,
$$

$\operatorname{Tr}_{H}\left(\widetilde{X^{\prime}}\right)-\mathfrak{C}=\left.(m-1) P\right|_{H}+\left.2 Q\right|_{H}+N_{3}+\cdots+N_{t}+Z_{m+1}+\cdots+Z_{s} \subset H \cong \mathbb{P}^{2}$.

Fix a generic line $L \subset H$. Consider the scheme $\widetilde{T}$ obtained from $\operatorname{Tr}_{H}\left(\widetilde{X^{\prime}}\right)-\mathfrak{C}$ by specializing the points $P, Q$ and the $m-3$ points $N_{3}, \ldots, N_{m-1}$ into $L$. By abuse of notation, we will again denote these specialized points by the same letters as before. Since $\operatorname{deg}(\widetilde{T} \cap L)=(m-1)+2+(m-3)=2 m-2$, the line $L$ is a fixed component for the curves of degree $2 m-3$ lying on $H$ and containing $\widetilde{T}$. Hence

$$
h^{0}\left(H, \mathcal{I}_{\widetilde{T}}(2 m-3)\right)=h^{0}\left(H, \mathcal{I}_{\operatorname{Res}_{L}(\widetilde{T})}(2 m-4)\right),
$$

where

$$
\operatorname{Res}_{L}(\widetilde{T})=\left.(m-2) P\right|_{H}+Q+N_{m}+\cdots+N_{t}+Z_{m+1}+\cdots+Z_{s} \subset H \cong \mathbb{P}^{2} .
$$


By applying Lemma 2.6 to the scheme $T^{\prime}$,

$$
T^{\prime}=\left.(m-2) P\right|_{H}+Z_{m+1}+\cdots+Z_{s} \subset H,
$$

which consists of one generic $(m-2)$-multiple point and $s-m=\left(\begin{array}{c}m-1 \\ 2\end{array}\right)-1$ generic 2 -dots, in degree $2 m-4$ we have

$$
\begin{aligned}
h^{0}\left(H, \mathcal{I}_{T^{\prime}}(2 m-4)\right) & =\max \left\{\left(\begin{array}{c}
2 m-2 \\
2
\end{array}\right)-\left(\begin{array}{c}
m-1 \\
2
\end{array}\right)-2(s-m), 0\right\} \\
& =\max \left\{\left(\begin{array}{c}
2 m-2 \\
2
\end{array}\right)-\left(\begin{array}{c}
m-1 \\
2
\end{array}\right)-2\left(\begin{array}{c}
m-1 \\
2
\end{array}\right)+2,0\right\} \\
& =\max \left\{\left(\begin{array}{c}
m \\
2
\end{array}\right)+2,0\right\} \\
& =\left(\begin{array}{c}
m \\
2
\end{array}\right)+2 .
\end{aligned}
$$

Since $\operatorname{Res}_{L}(\widetilde{T})$ is formed by the scheme $T^{\prime}$ plus the points $Q, N_{m}, \ldots, N_{t}$, which are $t-m+2=\left(\begin{array}{c}m+1 \\ 2\end{array}\right)-m+2=\left(\begin{array}{c}m \\ 2\end{array}\right)+2$ generic points, it immediately follows

$$
h^{0}\left(H, \mathcal{I}_{\operatorname{Res}_{L}(\widetilde{T})}(2 m-4)\right)=0,
$$

and from here by (14) we get

$$
h^{0}\left(H, \mathcal{I}_{\widetilde{T}}(2 m-3)\right)=0,
$$

that by the semicontinuity of cohomology gives

$$
h^{0}\left(H, \mathcal{I}_{\operatorname{Tr}_{H}\left(\widetilde{X^{\prime}}\right)-\mathfrak{C}}(2 m-3)\right)=0,
$$

so we are done.

5.3. Case $\mathbf{d} \equiv \mathbf{2}(\bmod 3)$. In this case we have, $($ see $(\ddagger))$,

$$
m=\frac{d+1}{3} ; \quad s=\left(\begin{array}{c}
m \\
2
\end{array}\right) ; \quad t=\left(\begin{array}{c}
m+2 \\
2
\end{array}\right)-1 .
$$

Note that $t \geq m$ by the definition. Now let $\widetilde{X^{\prime}}$ be the scheme obtained from $X^{\prime}$ by degenerating $m$ of the lines $L_{i}$ in such a way that they become a $(2, m)$-cone configuration $\widehat{\mathfrak{C}}$

$$
\widehat{\mathfrak{C}}=\mathfrak{C}+\mathfrak{D}_{H, m}(R),
$$

where $\mathfrak{C}$ is a cone configuration of type $m$ in $H$ and $R$ is the singular point of $\mathfrak{C}$. The remaining lines $L_{i}$, which are $t^{\prime}=t-m=\left(\begin{array}{c}m+2 \\ 2\end{array}\right)-1-m=\left(\begin{array}{c}m+1 \\ 2\end{array}\right)$ lines, are generic not lying on $H$, and we denote them by $L_{1}, \ldots, L_{t^{\prime}}$. So we get

$$
\widetilde{X^{\prime}}=\left.(m-1) P\right|_{H}+L_{1}+\cdots+L_{t^{\prime}}+\widehat{\mathfrak{C}}+C_{1}+\cdots+C_{s} \subset \mathbb{P}^{3}
$$


If we prove that $h^{0}\left(\mathcal{I}_{\widetilde{X^{\prime}}}(d-1)\right)=0$, then by the semicontinuity of cohomology we have $h^{0}\left(\mathcal{I}_{X^{\prime}}(d-1)\right)=0$, and we are done. In order to show that $h^{0}\left(\mathcal{I}_{\widetilde{X^{\prime}}}(d-1)\right)=0$, by Castelnuovo's inequality we need to show that

$$
h^{0}\left(\mathcal{I}_{\operatorname{Res}_{H}\left(\widetilde{X^{\prime}}\right)}(d-2)\right)=0 ; \quad h^{0}\left(H, \mathcal{I}_{\operatorname{Tr}_{H}\left(\widetilde{X^{\prime}}\right)}(d-1)\right)=0
$$

First we treat the residual of the scheme $\widetilde{X^{\prime}}$ with respect to the plane $H$. Since $\operatorname{Res}_{H}\left(\left.(m-1) P\right|_{H}\right)=\emptyset$, moreover $\operatorname{Res}_{H}(\widehat{\mathfrak{C}})=\left.(m-1) R\right|_{H}$, we have

$$
\operatorname{Res}_{H}\left(\widetilde{X^{\prime}}\right)=L_{1}+\cdots+L_{t^{\prime}}+\left.(m-1) R\right|_{H}+C_{1}+\cdots+C_{s} \subset \mathbb{P}^{3}
$$

Notice that $t^{\prime}=\left(\begin{array}{c}m+1 \\ 2\end{array}\right)$ and $s=\left(\begin{array}{c}m \\ 2\end{array}\right)$, then it is straightforward to see that $\operatorname{Res}_{H}\left(\widetilde{X^{\prime}}\right)$ is of the type $X_{\left(m, s, t^{\prime} ; d-1\right)}^{\prime}$ as in $\mathbf{H}_{d-2}^{\prime}$ for the case of $d-1 \equiv 1(\bmod$ 3 ), (one can check this by substituting $d-1$ in line 2 of $(\ddagger)$ ). Thus using the induction hypothesis yields the desired vanishing

$$
h^{0}\left(\mathcal{I}_{\operatorname{Res}_{H}\left(\widetilde{X^{\prime}}\right)}(d-2)\right)=0
$$

Now we are left with the trace scheme. Observe that the degenerate conic $C_{i}$ meets $H$ at the point $Q_{i}$, but $\operatorname{deg}\left(C_{i} \cap H\right)=2$. This observation is equivalent to saying that the trace of $C_{i}$ on $H$ is a 2-dot with support at $Q_{i}$. Let $Z_{i}$ denote this 2 -dot $(1 \leq i \leq s)$. Let $N_{i}=L_{i} \cap H,\left(1 \leq i \leq t^{\prime}\right)$. We get

$$
\operatorname{Tr}_{H}\left(\widetilde{X^{\prime}}\right)=\left.(m-1) P\right|_{H}+N_{1}+\cdots+N_{t^{\prime}}+\mathfrak{C}+Z_{1}+\cdots+Z_{s} \subset H \cong \mathbb{P}^{2}
$$

Because the cone configuration $\mathfrak{C}$ of type $m$ is a fixed component for the curves of $H^{0}\left(H, \mathcal{I}_{\operatorname{Tr}_{H}\left(\widetilde{X^{\prime}}\right)}(d-1)\right)$, removing $\mathfrak{C}$ implies that

$$
h^{0}\left(H, \mathcal{I}_{\operatorname{Tr}_{H}\left(\widetilde{X^{\prime}}\right)}(d-1)\right)=h^{0}\left(H, \mathcal{I}_{\operatorname{Tr}_{H}\left(\widetilde{X^{\prime}}\right)-\mathfrak{C}}(d-1-m)\right) .
$$

Hence our goal will be to prove

$$
h^{0}\left(H, \mathcal{I}_{\operatorname{Tr}_{H}\left(\widetilde{X^{\prime}}\right)-\mathfrak{C}^{(}}(d-1-m)\right)=0,
$$

that can be obtained in a simple way as follows.

Consider

$$
T=\left.(m-1) P\right|_{H}+Z_{1}+\cdots+Z_{s} \subset H,
$$

which is made by one generic $(m-1)$-multiple point and $s$ generic 2-dots. First we apply Lemma 2.6 to the scheme $T$ in degree $d-1-m$, which gives 
(recall that $d=3 m-1$ and $s=\left(\begin{array}{c}m \\ 2\end{array}\right)$ ),

$$
\begin{aligned}
h^{0}\left(H, \mathcal{I}_{T}(d-1-m)\right) & =\max \left\{\left(\begin{array}{c}
d-m+1 \\
2
\end{array}\right)-\left(\begin{array}{c}
m \\
2
\end{array}\right)-2 s, 0\right\} \\
& =\max \left\{\left(\begin{array}{c}
2 m \\
2
\end{array}\right)-\left(\begin{array}{c}
m \\
2
\end{array}\right)-2\left(\begin{array}{c}
m \\
2
\end{array}\right), 0\right\} \\
& =\max \left\{\left(\begin{array}{c}
m+1 \\
2
\end{array}\right), 0\right\} \\
& =\left(\begin{array}{c}
m+1 \\
2
\end{array}\right),
\end{aligned}
$$

next by the fact that $\operatorname{Tr}_{H}\left(\widetilde{X^{\prime}}\right)-\mathfrak{C}$ is formed by $T$ plus the points $N_{1}, \ldots, N_{t^{\prime}}$, which are $t^{\prime}=\left(\begin{array}{c}m+1 \\ 2\end{array}\right)$ generic points in $H$, we immediately get

$$
h^{0}\left(H, \mathcal{I}_{\operatorname{Tr}_{H}\left(\widetilde{X^{\prime}}\right)-\mathfrak{C}^{(}}(d-1-m)\right)=0 .
$$

Now the proof is complete.

\section{ProOF OF $\mathbf{H}_{d}^{\prime \prime}$}

This section is devoted to proving the statement $\mathbf{H}_{d}^{\prime \prime}$ for all $d \geq 3$, that is:

$\left(\mathbf{H}_{d}^{\prime \prime}\right): \quad$ Let $d \geq 3$, and

$$
\begin{gathered}
r=\left\lfloor\frac{\left(\begin{array}{c}
d+3 \\
3
\end{array}\right)}{d+1}\right\rfloor ; \quad q=\left(\begin{array}{c}
d+3 \\
3
\end{array}\right)-r(d+1) ; \\
m=\left\lfloor\frac{d}{3}\right\rfloor+1 ; \quad s=\left(\begin{array}{c}
m \\
2
\end{array}\right)+(r-m) d-\left(\begin{array}{c}
d+2 \\
3
\end{array}\right) ; \quad t=r-m-2 s .
\end{gathered}
$$

Let the scheme $X^{\prime \prime} \subset \mathbb{P}^{2}$ be the generic union of a cone configuration $\mathfrak{C}$ of type $m$; s double points $2 Q_{1}, \ldots, 2 Q_{s}$; $t$ simple points $N_{1}, \ldots, N_{t}$; and $q$ points $P_{1}, \ldots, P_{q}$ lying on a generic line $M$, i.e.

$$
X^{\prime \prime}=\mathfrak{C}+2 Q_{1}+\cdots+2 Q_{s}+N_{1}+\cdots+N_{t}+P_{1}+\cdots+P_{q} \subset \mathbb{P}^{2} .
$$

Then

$$
h^{0}\left(\mathcal{I}_{X^{\prime \prime}}(d)\right)=0
$$

Observe that the sections of $\mathcal{I}_{X^{\prime \prime}}(d)$ correspond to curves which have the cone configuration $\mathfrak{C}$ of type $m$ as a fixed component. Taking away $\mathfrak{C}$, we get

$$
h^{0}\left(\mathcal{I}_{X^{\prime \prime}}(d)\right)=h^{0}\left(\mathcal{I}_{X^{\prime \prime}-\mathfrak{C}}(d-m)\right)
$$

(note that by the definition we always have $d \geq m$, see $(\ddagger)$ ). 
To prove $h^{0}\left(\mathcal{I}_{X^{\prime \prime}-\mathfrak{C}}(d-m)\right)=0$, we proceed by considering the two cases $d \equiv 0,1(\bmod 3)$ simultaneously, and the case $d \equiv 2(\bmod 3)$ separately.

6.1. Case $\mathbf{d} \equiv \mathbf{0}, \mathbf{1}(\bmod \mathbf{3})$. From $(\ddagger)$ we have $q=0$, hence

$$
X^{\prime \prime}-\mathfrak{C}=2 Q_{1}+\cdots+2 Q_{s}+N_{1}+\cdots+N_{t} \subset \mathbb{P}^{2} .
$$

Since the points $N_{i}$ are $t$ generic simple points, to get $h^{0}\left(\mathcal{I}_{X^{\prime \prime}-\mathfrak{C}}(d-m)\right)=0$ it suffices to show that

$$
h^{0}\left(\mathcal{I}_{2 Q_{1}+\cdots+2 Q_{s}}(d-m)\right)=t .
$$

On the other hand, since the double point $2 Q_{i}$ imposes 3 independent conditions on the linear system of curves of given degree in $\mathbb{P}^{2}$, the expected value for $h^{0}\left(\mathcal{I}_{2 Q_{1}+\cdots+2 Q_{s}}(d-m)\right)$ is

$$
\max \left\{\left(\begin{array}{c}
d-m+2 \\
2
\end{array}\right)-3 s, 0\right\} .
$$

An easy computation by using $(\ddagger)$ yields

for $d \equiv 0(\bmod 3):(16)$ is equal to $\left(\begin{array}{c}m+2 \\ 2\end{array}\right)-3$, that is $t$;

for $d \equiv 1(\bmod 3)$ : (16) is equal to $\left(\begin{array}{c}m+1 \\ 2\end{array}\right)$, that is $t$.

Thus the equality (15) means that the scheme $\left(2 Q_{1}+\cdots+2 Q_{s}\right) \subset \mathbb{P}^{2}$ has good postulation in degree $d-m$.

From Corollary 2.5, which is an immediate consequence of the celebrated theorem by Alexander and Hirschowitz (Theorem 2.4), we know that apart from the generic union of two double points in degree 2 and generic union of 5 double points in degree 4 , all generic unions of double points in $\mathbb{P}^{2}$ have good postulation in a given degree. Now in our situation, one easily checks directly (by using $(\ddagger)$ ) that the generic union of $s$ double points in degree $d-m$ is not the exceptional cases of Corollary 2.5.

6.2. Case $\mathbf{d} \equiv \mathbf{2}(\bmod 3)$. From $(\ddagger)$ we have $q=m$, which implies that

$$
X^{\prime \prime}-\mathfrak{C}=2 Q_{1}+\cdots+2 Q_{s}+N_{1}+\cdots+N_{t}+P_{1}+\cdots+P_{m} \subset \mathbb{P}^{2} .
$$

Recall that

$$
m=\frac{d+1}{3} ; \quad s=\left(\begin{array}{c}
m \\
2
\end{array}\right) ; \quad t=\left(\begin{array}{c}
m+2 \\
2
\end{array}\right)-1 .
$$

Now we have $d-m=2 m-1$. For the purpose of having $h^{0}\left(\mathcal{I}_{X^{\prime \prime}-\mathfrak{C}}(2 m-1)\right)=$ 0 , we make a specialization of the scheme $X^{\prime \prime}-\mathfrak{C}$ via the line $M$, which already contains the points $P_{1}, \ldots, P_{m}$. Let $\widetilde{X^{\prime \prime}}$ be the scheme obtained from $X^{\prime \prime}-\mathfrak{C}$ by specializing the points $N_{1}, \ldots, N_{m}$ into the line $M$ (note that this is possible because $t>m$ by the construction). If we show that

$$
h^{0}\left(\mathcal{I}_{\widetilde{X^{\prime \prime}}}(2 m-1)\right)=0
$$


then by the semicontinuity of cohomology we are done.

Because of the observation that $\operatorname{deg}\left(\widetilde{X}^{\prime \prime} \cap M\right)=m+m=2 m$, the line $M$ is a fixed component for the curves of degree $2 m-1$ containing $\widetilde{X^{\prime \prime}}$. Hence

$$
h^{0}\left(\mathcal{I}_{\widetilde{X^{\prime \prime}}}(2 m-1)\right)=h^{0}\left(\mathcal{I}_{\operatorname{Res}_{M}\left(\widetilde{X^{\prime \prime}}\right)}(2 m-2)\right),
$$

where

$$
\operatorname{Res}_{M}\left(\widetilde{X^{\prime \prime}}\right)=2 Q_{1}+\cdots+2 Q_{s}+N_{m+1}+\cdots+N_{t} \subset \mathbb{P}^{2} .
$$

A quite simple consideration shows that the scheme $\left(2 Q_{1}+\cdots+2 Q_{s}\right)$ in degree $2 m-2$ is never in the exceptional cases of Corollary 2.5. Therefore it has good postulation, i.e.

$$
\begin{aligned}
h^{0}\left(\mathcal{I}_{2 Q_{1}+\cdots+2 Q_{s}}(2 m-2)\right) & =\max \left\{\left(\begin{array}{c}
2 m \\
2
\end{array}\right)-3 s, 0\right\} \\
& =\max \left\{\left(\begin{array}{c}
2 m \\
2
\end{array}\right)-3\left(\begin{array}{c}
m \\
2
\end{array}\right), 0\right\} \\
& =\max \left\{\left(\begin{array}{c}
m+1 \\
2
\end{array}\right), 0\right\} \\
& =\left(\begin{array}{c}
m+1 \\
2
\end{array}\right) .
\end{aligned}
$$

By using the fact that the points $N_{m+1}, \ldots, N_{t}$ are $t-m=\left(\begin{array}{c}m+2 \\ 2\end{array}\right)-1-m=$ $\left(\begin{array}{c}m+1 \\ 2\end{array}\right)$ generic points in $\mathbb{P}^{2}$, the above equality gives

$$
h^{0}\left(\mathcal{I}_{\operatorname{Res}_{M}\left(\widetilde{X^{\prime \prime}}\right)}(2 m-2)\right)=0,
$$

which finishes the proof.

\section{REFERENCES}

[AB14] T. Aladpoosh, E. Ballico, Postulation of disjoint unions of lines and a multiple point, Rend. Sem. Mat. Univ. Politec. Torino. 72 (3-4) (2014), 127-145.

[Ala16] T. Aladpoosh, Postulation of generic lines and one double line in $\mathbb{P}^{n}$ in view of generic lines and one multiple linear space, arXiv:1606.02974 [math.AG] (2016).

[AH95] J. Alexander, A. Hirschowitz, Polynomial interpolation in several variables, J. Alg. Geom. 4 (2) (1995), 201-222.

[AH00] J. Alexander, A. Hirschowitz, An asymptotic vanishing theorem for generic unions of multiple points, Invent. Math. 140 (2) (2000), 303-325.

[Bal11] E. Ballico, Postulation of disjoint unions of lines and a few planes, J. Pure Appl. Algebra, 215 (4) (2011), 597-608.

[Bal15] E. Ballico, Postulation of disjoint unions of lines and a multiple point II, Mediterr. J. Mat. (2015), 1-15.

$\left[\mathrm{BDS}^{+} 17\right]$ T. Bauer, S. Di Rocco, D. Schmitz, T. Szemberg, J. Szpond, On the postulation of lines and a fat line, arXiv:1706.02350 [math.AG] (2017).

[CCG10] E. Carlini, M. V. Catalisano, A. V. Geramita, Bipolynomial Hilbert functions, J. Algebra. 324 (4) (2010), 758-781. 
[CCG11] E. Carlini, M. V. Catalisano, A. V. Geramita, 3-dimensional sundials, Cent. Eur. J. Math. 9 (5) (2011), 949-971.

[CCG16] E. Carlini, M. V. Catalisano, A. V. Geramita, On the Hilbert function of lines union one non-reduced point, Ann. Sc. Norm. Super. Pisa Cl. Sci (5) XV (2016), 69-84.

[CV11] E. Carlini, A. Van Tuyl, Star configuration points and generic plane curves, Proc. Am. Math. Soc. 139 (12) (2011), 4181-4192.

[CG94] M. V. Catalisano, A. Gimigliano, On curvilinear subschemes of $\mathbb{P}^{2}$, J. Pure Appl. Algebra. 93 (1994), 1-14.

[CoC04] CoCoATeam, CoCoA: a system for doing computations in Commutative Algebra, available at http://cocoa.dima.unige.it, 2004.

[Ful84] W. Fulton, Intersection theory, Springer-Verlag, Berlin Heidelberg New York, 1984.

[GMR83] A. V. Geramita, P. Maroscia, L. G. Roberts, The Hilbert function of a reduced k-algebra, J. Lond. Math. Soc. (2) 28 (3) (1983), 443-452.

[Har77] R. Hartshorne, Algebraic geometry, Springer-Verlag, New York, 1977.

[HH81] R. Hartshorne, A. Hirschowitz, Droites en position générale dans l'espace projectif, in: Algebraic Geometry, La Rábida, (1981); in: Lecture Notes in Math, vol. 961, Springer, Berlin, (1982), 169-188.

[Hir81] A. Hirschowitz, Sur la postulation générique des courbes rationnelles, Acta Math. 146 (1981), 209-230.

Tahereh Aladpoosh; School of Mathematics, Institute for Research in Fundamental Sciences (IPM), P.O.Box: 19395-5746, Tehran, Iran.

E-mail address: tahere.alad@ipm.ir

Maria Virginia Catalisano; Dipartimento di Ingegneria Meccanica, Energetica, Gestionale e dei Trasporti, Università degli Studi di Genova, Genoa, ITALY.

E-mail address: catalisano@dime.unige.it 